\title{
Mesoscopic collective dynamics in liquids and the Dual Model
}

Fabio Peluso ( $\sim$ fpeluso65@gmail.com )

Finmeccanica: Leonardo SpA https://orcid.org/0000-0003-0890-9772

\section{Research Article}

Keywords: Liquid model, Phonons in liquids, Mesoscopic model of liquids, phonon - particle interaction, Relaxation time

Posted Date: February 19th, 2021

DOI: https://doi.org/10.21203/rs.3.rs-205220/v1

License: (9) This work is licensed under a Creative Commons Attribution 4.0 International License. Read Full License 


\title{
Mesoscopic collective dynamics in liquids and the Dual Model.
}

\author{
Fabio Peluso \\ Leonardo SpA - Electronics Division - Defense Systems LoB \\ Via Monterusciello 75, 80078 Pozzuoli (NA) - Italy \\ mailto:fpeluso65@gmail.com.
}

\begin{abstract}
.
In the present article it is shown how a series of experimental evidences and theoretical developments on liquid modelling, gathered for the first time, can all be framed in a mesoscopic view of liquids that are hypothesized as constituted by a population of wave packets, responsible for the propagation of elastic and thermal perturbations, and of dynamic aggregates of molecules, in continuous re-arrangement, diving in an ocean of amorphous, disordered liquid. This model, dubbed Dual Model of Liquids, is complementary to the Phonon theory of Liquid Thermodynamics, recently proposed by an independent group. The pseudo-crystalline dynamic structures, whose presence in liquids is evidenced by high energy inelastic scattering experiments, interact with a statistical population of harmonic elastic waves and anharmonic wave-packets propagating within and among the structures themselves, respectively. The expression for the interaction term is derived from "first principles" based on general considerations related to the pressure exerted by elastic waves travelling in condensed media. The anharmonic character of the interaction allows the exchange not only of energy but also of momentum between wave packets and clusters, thus determining both the displacement of the latter within the medium, and the redistribution of the energy between external, or translatory degrees of freedom of the clusters, and internal collective, vibratory degrees. Using these concepts it is possible to calculate some dynamic and thermodynamic quantities concerning the dynamics of liquids. Moreover, the interpretation of the relaxation times of the processes involved in momentum and energy transport is given, their Order-of-Magnitude is calculated, and the way in which these times are involved in the different phases of the collective dynamics of liquids is discussed. A comparison is provided with results obtained in the frame of PLT and with the forecasts for the visco-elastic transition regions.
\end{abstract}

In the last part of the paper, some experiments are suggested that should be performed to provide additional details to the model.

Keywords: Liquid model, Phonons in liquids, Mesoscopic model of liquids; phonon - particle interaction; Relaxation time

\section{Introduction.}

Liquid and solid states are thermodynamic concepts correlated with the relative motions of the constituent molecules and the rates at which energy and momentum are redistributed among the available degrees of freedom (DoF). The three states of matter are characterized by very different behaviour, in particular that of liquid state is sometimes contradictory, and this got way confusion in the mind of physicists. Just to cite some, on macroscopic scale the molecules of solids are strongly bounded to their positions for long times, while liquids, as gases, besides incapable of opposing to tangential stresses, are also characterized by diffusive phenomena evolving on short timescales. On the 
other hand, fusion is accompanied by a relatively small amount of volume, by $10 \%$ at maximum, while the passage from liquid to vapour and gas states is characterized by a huge increase of specific volume. On microscopic scale intermolecular distances are about the same in solids and liquids and the latent heat of fusion is much smaller than the latent heat of vaporization. These facts allow us to affirm that binding energies are similar in solids and liquids, while in liquids long-range order is lost and local order reduced, making a liquid isotropic, or amorphous, like a gas.

On a macroscopic scale liquids are being studied with the methods of fluid dynamics, while on a microscopic scale it is obvious to resort to those of quantum mechanics. Already in XIX century it was realized that fluid dynamics was not able to explain what was considered an anomalous behaviour of liquids compared to that expected for a condensed gas, as they were initially classified according to their macroscopic behaviour. The advent of quantum mechanics then paved the way towards liquid models seen as "molten solids". This allowed us to realize that it became necessary to investigate an intermediate, mesoscopic scale for liquids. On this intermediate scale the behaviour of liquids was the object of theoretical investigations originally proposed by Debye [1,2], Brillouin [3,4], and Frenkel [5]. They faced the theoretical study from different approaches, although all of them contributed to the global vision of liquid structure that emerged later from the experimental investigations over mesoscopic scale. On this scale, liquids deviate from their macroscopic behaviour, although the dynamics still involve a large number of molecules. The relaxation mechanisms become fundamental in the dynamics of molecules in liquids and in the processes of redistribution of energy and momentum among the available DoF. Due to the complexity of liquid behaviour on different scales, a general and unifying physical modelling of liquid structure is still an open question of modern physics. Experimental results obtained in the last forty years allow to demonstrate the validity and limits of the approach adopted in hydrodynamic fluctuation modelling (many references may be found in [6]), and to advance new insights on the mesoscopic structure of liquids [7-12].

As is well known, at low frequencies, i.e. large wavelengths, it is not possible to "see" the mesoscopic structure of liquids. The entire liquid oscillates, compressing and expanding under the effect of pressure waves, which travel at the speed of sound. However, when one investigates the behaviour of a liquid at very high frequencies, that is at small wavelengths, one discovers that it has a mesoscopic structure organized on dynamic solid-like clusters, also called elsewhere solid-like or pseudo-crystalline structures [see for instance 7-37] (we will call them here also in a more picturesque and imaginative way with the term "dynamic icebergs"). Around mid 60's the study of the static structure factor $S(q)$, obtained by means of Inelastic Neutron Scattering (INS) and Inelastic X-ray Scattering (IXS), showed that in liquids, and especially in water, it was characterized by the presence of elastic contributions that, in turn, contributed an enhancement of the $S(q)$ profiles at high frequencies. In particular, Sköld [38] interpreted the $S(q)$ as a measure of the effective number of atoms contributing to the scattered intensity at a given exchanged wave vector $q$. This argument was recovered years later $[12,10]$ when the IXS scattering benefited of advanced techniques able to perform experiments in the THz regions of the perturbing spectrum. It became then natural to ascribe the $q$ dependence of the scattered spectra on the higher inertia of the target system due to the large number of atoms participating to the collective response of the target system. In particular, the first neighbour movements become more correlated when $q$ matches the inverse of the first neighbour's separations and $S(q)$ approaches its maximum [12]. The forerunner and most surprising experimental datum on the dynamics of liquids at high frequencies is that of Ruocco and Sette [15]. In 1996 they measured by IXS experiments, therefore at high frequency, in liquid water at ambient conditions, the propagation speed of elastic waves, and found it equal to $3200 \mathrm{~m} / \mathrm{s}$, i.e. about double that known at traditional frequencies, $1500 \mathrm{~m} / \mathrm{s}$, and very close to that of solid water, $4000 \mathrm{~m} / \mathrm{s}$. Starting from the 
experimental results obtained in various liquids with IXS techniques, [see for example 8-24], the evidence that pseudocrystalline structures "exist and persist" in liquids has gradually consolidated, their size and number depending on the liquid temperature (and pressure). These structures can obviously be discovered only when high frequency measurements are carried out, typical of INS and IXS techniques, the wavelengths of the radiation involved being small enough to reveal their presence. In the Dual Model of Liquids (DML) framework, dealt with in this paper, these pseudo-crystalline structures interact with the rest of the (amorphous) liquid by means of anharmonic interactions which arise at their border, a quasi-elastic propagation within the clusters is transmitted to the amorphous matrix in the form of anharmonic wave-packets, and vice versa. This idea is depicted in pictorial way in Figure 1. Unlike in crystalline solids, the anharmonicity allows a non-infinitesimal duration of the interactions, with consequent transport not only of energy but also of momentum, and furthermore exchange of energy with the internal DoF of the solid-like dynamic structures. Since this dynamics occurs mainly at high frequencies, and involves only the DoF of the lattice, the parameter $m$ has been introduced (see Eq. (15)) to account for the average number of DoF actually participating to the collective dynamics. Therefore, the model provides an intuitive way of describing liquids how they emerge from the experiments. On the other hand, it would not be surprising if the transition from the solid to the liquid state does not suddenly occur in the narrow range of the triple point, but rather over a much wider range of temperature and pressure, this fact being the explanation of the "existence and persistence" of pseudo-crystalline structures inside the liquids above the melting point, as found in the IXS experiments performed by several groups.

Frenkel and Brillouin had not gone so far as to model the interaction mechanism and to write the related interaction term between elastic waves and molecule clusters, as well as to provide the basis for writing the thermodynamic and fluid dynamic coefficients. Frenkel himself admits, in the introduction of his book, that the kinetic theory of the liquids (he proposes), even being in a very crude and incomplete form, may serve to attract the attention of other scientists to this subject and accelerate its further development [5]. Recent experimental discoveries however [8-25] have given credit again to their early intuitions. The solid-like approach for the mesoscopic liquid state pushed physicists to re-frame all the theoretical bases developed until then. This was made possible by the evidence of rigidity and short-range order in liquids on one hand, and elements of fluidity and disorder in solids, on the other. The kinetic theory of liquids was therefore developed as an extension of solid models. The ideas of Debye, Brillouin and Frenkel were not considered for about 40 years, until Fiks used them again in the early " 60 s to explain thermal diffusion in liquids [39]. In his work Fiks derived an expression for the coefficient of thermal diffusion, i.e. with a temperature gradient externally applied to the system. Subsequently Fiks applied the expressions arrived at to the electronic drag and thermal diffusion in metals [40]. In a very interesting paper Andreev [41] assumes that a liquid consists of two weakly coupled systems, the phonons and the remainder of the liquid. He calculates the effects connected with the presence of weakly damped phonons in a normal liquid. In particular, he provides the expressions for mechanothermal and thermo-mechanical effects, as well as the propagation of shear oscillations. Similar ideas were later developed and extensively applied by Gaeta and co-workers to describe the behavior of non-isothermal liquids, allowing to interpret some of their typical phenomena, such as thermo-osmosis, thermodialysis and the Soret effect [42-46]. In particular the model provided the heat diffusion direction of the solute, or of the solvent, with respect to that of the applied temperature gradient.

All the models described above have a single common leitmotif, that is the presence of a temperature (or concentration) gradient externally applied to a liquid solution. In the present paper, however, this limiting constraint is completely removed and overtaken; an interaction model is built between wave-packets and the clusters of liquid molecules of a pure liquid, regardless on whether external gradients are applied to the system. The cue for this insight comes from the experimental evidences cited above that have indirectly confirmed the presence and permanence of 
pseudo-crystalline dynamic structures in liquids. Besides, and maybe more relevant, is the fact that DML also allows to identify and quantify, at least the Order of Magnitude (OoM) of the most relevant relaxation times which characterize the dynamics of the interaction process occurring in the liquid at mesoscopic scale, between the "liquid particles", (the clusters of molecules, in the general meaning of a dynamic aggregate of molecules) and the "lattice particles", i.e. the elastic wave-packets. A peculiarity of the interaction term between lattice particles and liquid particles is its anharmonic character, that allows the exchange not only of energy but also of momentum between wave packets and clusters, thus determining both the displacement of the latter within the medium, and the redistribution of the energy between external, or translatory DoF of the clusters, and internal collective, vibratory DoF.

Further implications and results of the DML are illustrated in what follows. In a series of recent papers [7,25-34] an independent group has proposed the Phonon theory of Liquid Thermodynamics (PLT). They introduce an interacting Hamiltonian for describing the dynamics of a phonon field. In a similar frame as that of DML, in PLT the system is substantially considered as made of two interacting subsystems [28]; indeed Authors start from a solid-like expression of the Hamiltonian in harmonic approximation, i.e. without interaction, that they extend to consider anharmonic contributions by introducing an appropriate interacting term [7,32]. This approach has many interesting results. First, it can provide a unique expression of the Hamiltonian for the three states of matter, solid, liquid and gas. Second, it provides for liquids two distinct solutions, depending on the value of the frequency: for frequencies lower than a specific value, that the Authors of PLT identifies with the Frenkel frequency $\omega<\omega_{F}$, the system is characterized by one longitudinal mode, while for frequencies higher than $\omega_{F}$, the system exhibits three modes, namely two transverse non massive (i.e. that do not involve mass transport) and one longitudinal massive, (i.e. that involves mass transport). Among the results achieved with PLT, one that deserves mention is the theoretical expression for the specific heat $C_{V}$, that has been validated in 21 different liquids [25]. The expression arrived at covers the cases of the solid, glassy, liquid, gas and quantum liquids states of matter, giving for $C_{V}$ a theoretical limit value of $3 R$ for solids down to $2 R$ for liquids $[7,29,32]$. Another relevant result of the DML, and that compares with PLT, is that it provides an expression for $C_{V}$ as function of the number of excited collective DoF. We will return on the calculation of $C_{V}$ in Section 3.3, where an expression for $C_{V}$ is determined in the frame of DML. It is however important to highlight since now that the DML proposed here is not in contrast with the PLT, on the contrary they face the problem of liquid model from two different and complementary approaches, as more clearly specified in the Discussion. The elementary interaction between wave-packets and liquid particles, together with the developments deriving from the model, allow to determine the contribution to some fundamental parameters characterizing the liquid medium due to these interactions, such as thermal conductivity, specific heat, diffusion coefficient, molecule drift velocity, etc.. An apparently simplifying hypothesis is that the collisions between wave packets and pseudo-crystalline structures have a single-particle character, as in the Boltzmann-Maxwell statistics. This hypothesis is however supported by the calculation of the density of wave packets (see section 3.4), which appears to be of the same OoM as the density of molecules, if not lower.

Before going on to describe the model here proposed of mesoscopic structure of liquids, it is worth pointing out right away that it does not want to be in contrast with those derived from the continuum mechanics but, starting from the experimental evidences, it provides a possible vision of how liquids are organized at microscopic (or rather mesoscopic) scale. The model therefore has no claim to rewrite pages of physics that are perfectly framed by continuum mechanics. As well assessed experimentally, the classical hydrodynamic theory for continuous media 
consistently describes the dynamics of density fluctuations in simple liquids at quasi-macroscopic distances and timescales. This theoretical approach provides the so-called Dynamic Structure Factor, $S(k, \omega)$. When discussing the evolution of the $S(k, \omega)$ shape across various dynamic regions, it is useful to take the average inter-atomic separation $d$ and mean inter-collision time $\tau_{\text {coll }}$ as references, and compare them with the probed distance, $l=2 \pi / k$ and time window, $t=2 \pi / \omega$, respectively. From experiments one may consider two distinct situations, namely [10-12]:

a) $l>>d$ and $t>>\tau_{\text {coll }}$, the system appears as a continuous and isotropic medium, its dynamic response is probed by averaging over a large number of microscopic interactions. In this limit, any detailed information on the microscopic structure and internal DoF of the sample is lost, and the hydrodynamic theory for continuous and homogeneous media gives a consistent account of the dynamic response.

b) In the opposite limit $l<<d$ and $t<<\tau_{\text {coll }}$ the probed dynamic event reduces to the free recoil of the single atom induced by the collision with the probe particle (e.g. a photon or a neutron) and before any successive interactions with first neighbouring atoms. Here, the shape of the spectrum mirrors the momentum distribution of the struck particle in its initial state, which, for a classical system, is determined by the Maxwell-Boltzmann distribution.

Although the $S(k, \omega)$ shape is well-known in these two limits, no sound theory predicts its evolution in between them. At these scales, thermodynamic and transport parameters are no longer constant coefficients, but rather space and time-dependent local variables of the fluid. To cover the gap, a suitable Generalized Hydrodynamics (GH) theory of density fluctuations has been developed. Along the crossover between the two limits, density fluctuations become strongly coupled with molecular DoF, making the interpretation of $S(k, \omega)$ particularly complex. Owing to the lack of a rigorous theory predicting such a Q-dependence, the various theoretical attempts to extend the hydrodynamic description at finite $\mathrm{Q}$ values mostly prescribe phenomenological recipes (and many of them do not provide a quantitative prediction of the Q-dependence of transport parameters). In particular the GH models rely on the assumption that the departure from the Rayleigh-Brillouin shape is smooth enough to leave the formal structure of the hydrodynamic spectrum unaltered, yet they assume that the relevant shape parameters have an unknown Qdependence. Such a Q-dependence is usually determined introducing some free parameters, whose amplitude is determined a posteriori by best fitting the GH model to the line-shapes measured (or computed) at various $\mathrm{Q}$ values. The memory functions introduced in GH are ad hoc mathematical expressions built with the aim of adapting the hydrodynamic equations and their solutions to the intermediate case of the viscoelastic regime of liquids. This theoretical framework is normally referred to as the "memory functions formalism", or also the Zwanzig-Mori formalism [47-48]. The approach used by GH and memory functions formalism are well known and largely used in physics. Using a modern way of saying, we could say that GH adopts a top-down approach.

The paper is organized as follows. In paragraph 2 early theoretical models are briefly recalled and compared with the results obtained in the recent scattering experiments on liquids. In particular, the role of the relaxation time is discussed. In paragraph 3 the DML is presented through the description of the thermal, or elastic, excitations, and of their interaction with the dynamic icebergs. In paragraph 3.2 it is shown how the expression of the interaction term can be deduced on the basis of "first principles" of wave mechanics. In paragraph 3.3 the dynamics of the interactions between wave packets and liquid particles is analyzed, and the expressions of thermal capacity, thermal conductivity, and in general of the contribution to the internal energy of the liquid due to the presence of wave packets are derived. Section 3.4 is dedicated to the step-by-step description of the interaction process and to identify the several relaxation times that characterize it, to calculate their OoM and finally to compare the results with the PLT and with the forecasts 
of the visco-elastic cross-over in liquids. In paragraph 4 the expressions obtained in paragraph 3 are discussed in detail and the results compared with the recent theoretical and experimental achievements on the structure of liquids at the mesoscopic level. The role of interaction and relaxation times is deeply analysed. Finally, in the "Conclusions" some experiments are proposed, among other things, to be conducted possibly also in the virtual absence of gravity to reveal and study the dynamic evolution of wave packets propagation in liquids.

\section{Early theoretical models and recent experimental evidences of collective dynamics in liquids.}

The hypothesis that thermodynamic equilibrium properties of liquids could be interpreted in terms of those of thermal excitations, supposed as consisting of high-frequency wave-packets, was first introduced by Debye [1,2], Brillouin [3,4], Frenkel [5] and Lucas [49]. Debye extended his theory of heat capacity of solids to the liquid state, neglecting transverse waves since liquids lack of rigidity. He assumed the longitudinal high-frequency waves to be very similar to the collective oscillations of solids. Thermal energy in a liquid is then equally distributed among the various DoF of the molecules and the phonons. Brillouin accepted the argument of Debye but observed that, neglecting transverse waves, the Debye model would have lead to the paradox of a higher limiting Debye frequency $v_{D}$ in liquids than in solids. A fundamental forecast of Brillouin [3,4], later reworked by Landau [50] and Fabelinskii [51], was that a light beam crossing a liquid would have been affected by a Doppler shift. When a scattering experiment is performed, a density fluctuation is induced in the system under investigation by the impinging probe. This (neutron or X-ray) is scattered by the target particle, giving rise to the spectroscopic pattern proportional to the dynamic structure factor, $S_{E Q}(k, \omega)$ [52-54], which is a function of the energy and momentum exchanged in the scattering event. This was confirmed for the first time some years later by Meyer and Ramm [55] and by RaghavendraRao [56]; they found experimental evidence indeed that light diffusion in liquids is accompanied by a frequency shift. The result is the emergence from the liquid of a Lorentian triplet, a central, unshifted line (Rayleigh), accounting for elastic scattering, and two side-lines (Brillouin doublet) symmetrically shifted with respect to the central one, accounting for inelastic scattering, the frequency shift being proportional to the sound speed in the liquid. More specifically [52], when a light beam impinges on a liquid volume, the emerging Rayleigh-Brillouin spectrum arises from the inelastic interaction between a photon and the hydrodynamic modes of the fluids. The doublet, named as the "Stokes" and "anti-Stokes" components of the translational spectrum of the liquid, is due to the inelastic collision between an impinging photon and the fluid in which the photon gains or loses energy to the phonons, or sound modes, in the fluids, and thus undergoes a frequency shift

$$
\text { 1. } \Delta \omega_{B}(k)= \pm c_{s} q
$$

where $c_{s}$ is the sound velocity in the liquid, $q$ the wave vector. The HWHM of these two lines gives the lifetime of a classical phonon of wave vector $q$, while that of the unshifted line gives the thermal diffusion coefficient of the liquid, $D_{T}=K / \rho C_{P}$. Provided that the three lines are well resolved, a suitable light scattering experiment may provide experimental evaluation of relevant liquid parameters.

Another important key point raised by Brillouin is the value of the specific heat of monatomic liquids, $C_{V}$. From a classical point of view, $C_{V}$ should increase with temperature from the melting point to the critical point; 
measurements show instead that it decreases from about $3 R$ at the melting point down to $2 R$ at the critical point, where $R$ is the gas constant [57]. The interpretation of this experimental evidence in the frame of the Debye model was provided by Brillouin [4] and Frenkel [5], by admitting that a pseudo-crystalline structure still survive in liquids above the melting point. More recently, the Phonon theory of Liquid Thermodynamics has provided a theoretical expression for $C_{V}$ in line with the experimental measurements [7,25-34].

There were no relevant advancements in the development of a liquid model until the end of the 70's - beginning of the 80 's (if one neglects the contribution of Fiks, Andreev and Gaeta cited before) when many papers begun to be published on the study of hydrodynamic fluctuations in liquids out of equilibrium. In particular, some Authors [58-64] calculated the form of the light spectrum scattered from a liquid near or far from thermal equilibrium. According to [58-62], in case of small gradients, by using perturbation theory around a state of total equilibrium, the line shape and intensity are modified with respect to the ordinary Landau-Placzek formula [50-51]. In particular, the central Rayleigh line remains the same as in equilibrium, while shape and intensity of the two Brillouin lines deviate from their equilibrium values by terms proportional to the temperature gradient. The dynamical structure factor for a fluid in nonequilibrium steady state (NE) with a small temperature gradient $(\mathrm{SG})$ is given by:

$$
\text { 2. } S_{N E}^{S G}(q, \omega)=S_{E Q}^{R}+\sum_{\sigma= \pm 1} S_{E Q}^{B}(\sigma)\left[1-\frac{\sigma y_{\sigma} c \hat{q} \cdot \nabla T}{\Gamma_{s} q^{2} T}\right]
$$

where $\hat{q}$ is the unitary wave-vector, $\sigma= \pm 1$ according to the Brillouin peaks, $\omega$ the frequency, $\Gamma_{s}=(4 v / 3+\zeta / \rho)+(\gamma-1) D_{T}$ the sound-damping constant (the product $\Gamma_{s} \cdot q^{2}$ is the linewidth), $c$ the speed of sound in the medium and finally $\left.y_{\sigma}=1+\frac{\mid\left(\Gamma_{s} q^{2} / 2\right)^{2}-(\omega-\sigma c q)^{2}}{\left[\left(\Gamma_{s} q^{2} / 2\right)^{2}+(\omega-\sigma c q)^{2}\right.}\right]$. As for the intensity of light scattered by the sample, although the total intensity remains unchanged, a dependence on the wave-vector $\hat{q}$ appears in the two Brillouin lines:

$$
\text { 3. } I_{N E}^{S G}=I_{E Q}^{R}+\sum_{\sigma= \pm 1} I_{E Q}^{B}\left(1-\frac{\sigma c \hat{q} \cdot \nabla T}{\Gamma_{s} q^{2} T}\right) .
$$

The analysis of Eqs. (2) and (3) leads to the following considerations. First, we note that the central line is not affected by the temperature gradient; second, although the positions of the Brillouin lines are unchanged, they are asymmetric and non-Lorentzian, the asymmetry factor being proportional to the ratio $\nabla T / q^{2}$. The same proportionality is found in the difference of intensity between the two Brillouin lines. According to [58-63], the $q^{-2}$ dependence in both shape and intensity indicates the presence of long-range correlations in the fluid out of thermal equilibrium, proportional to the inverse of the average intermolecular distance. Besides, the asymmetry in the Brillouin lines is due to a coupling between two sound modes with the heat current produced by the temperature gradient. Owing to this coupling, the compressional waves travelling in the same direction as the macroscopic heat flow will have a larger amplitude than waves travelling in opposite direction. We will return on this aspect when dealing with the elementary mechanism of phonon $\leftrightarrow$ liquid particle interaction at the base of the DML, and in the Discussion.

When large temperature gradients are applied to the liquid, shape and intensity also of the central Rayleigh line show deviations from equilibrium values, proportional to the square of the temperature gradient [62]. In particular, the 
intensity is enhanced when compared to its equilibrium value, while the deviation of intensities of the Brillouin lines from their equilibrium values has a rather complex dependence upon the temperature gradient.

The interest in calculating the non-equilibrium correlation functions was stimulated by the fact that, owing to the presence of the gradients in the system, such correlation functions have a much longer range in space than their equilibrium counterparts, and that these long-range non-equilibrium correlations could be detected by means of lightscattering techniques. The above theoretical works were motivated by the results found by Beysens and co-workers [65] who performed very-low-angle Brillouin light-scattering experiments in liquid water subjected to a temperature gradient. Their results showed the Brillouin asymmetry in shape and intensity foreseen by Kirkpatrick [61-62]. Later on, other very-low-angle light-scattering experiments were performed with various liquids far from critical conditions [66-68]. Experimental results confirmed the theoretical forecasts obtained with the non-equilibrium fluctuations approach, and the observed phenomenology of asymmetry in the Brillouin lines was interpreted as due to an asymmetry in the phonon flux induced by the temperature gradient [65-68]. Because Brillouin lines account for energy and momentum transferred in inelastic scattering events, and the central Rayleigh line accounts for elastic scattering, at large temperature gradients, while the correlation lengths still increase with respect to the isothermal or near to thermal equilibrium values [61-62], it is expected that inelastic contributions of phonon-lattice interactions also have a large relative importance in the overall dynamic processes.

So far the early theoretical forecasts of the presence of long-range correlations in liquids. Very interesting results came also from the numerical modelling of isothermal (equilibrium) liquids. In the seventies, an independent research group [69] set-up a numerical model to calculate $S_{E Q}(q, \omega)$ for liquid water based on the Molecular Dynamics approach. In their model, fluctuation phenomena were analysed occurring in a system of 216 water molecules kept at ambient steady conditions and at thermal equilibrium. Results indicated that transverse currents were present in the liquid in the form of propagating collective modes, and that the spectrum of density of fluctuations exhibited a secondary maximum at a much higher frequency than the normal frequency of sound propagation. Due to the difficulties of resolving the spectral lines in liquids at ordinary light frequencies, experimental verifications of the predictions of the numerical simulation of Rahman and Stillinger came first in the mid of eighties with the advent of INS technique [37]. Few years later, the advent of IXS techniques allowed confirming the previous experimental results with a higher order of resolution. By studying the Brillouin doublets generated when X-rays are scattered by the molecules of the substance under investigation, it was experimentally found that in isothermal liquids, besides the collective non dispersing mode propagating at the ordinary speed of sound, another branch exists of collective modes, linearly dispersing with the frequency and propagating at a speed higher than that of ordinary sound [see for instance 9-12, 13-24, 36]. In particular, for water the cross-over was detected between the ordinary speed of sound, $\approx 1500 \mathrm{~m} / \mathrm{s}$, and that of collective modes, with positive frequency dispersion, propagating at a speed more than twice that of ordinary sound, $\approx 3200 \mathrm{~m} / \mathrm{s}$. This result, already foreseen in [69], was correlated with the arrangement typical of liquid water at mesoscopic scale, marking a transition from ordinary to "fast" sound. The study of this transition as function of temperature and frequency has made possible to relate this phenomenon to a structural relaxation process, which shows many analogies with the glass-forming systems $[8,24]$.

The occurrence of a solid-like value of sound propagation in liquids can be theoretically explained also following the points of view of Brillouin and Frenkel. Indeed we may distinguish two limit regimes for propagation of perturbations in liquids. One is the "normal" hydrodynamic regime, in which one considers the medium as a continuum and the excitations on a so long timescale that the system may be assumed to be in thermodynamic 
equilibrium. This regime is also usually referred to as viscous. The second is the "solid-like" or elastic regime, in which the dynamics becomes that of a free particle between successive elastic collisions. The investigation of collective dynamics becomes of particular interest when intermediate time- and length-scale are considered, i.e. distances comparable to those characterizing the structural correlations among particles, and times comparable to the lifetimes of such correlations. Although these two extreme behaviours, viscous and elastic, are well known in physics, the intermediate situation is still well far from being fully characterized. This intermediate range is referred to as viscoelastic regime. In the Discussion this topic is further developed.

The value obtained experimentally for the speed of sound in liquid water [13-15,20] proves that, in the range of momentum exchange examined and during the time lapse of the relaxation time, water behaves as a rigid network of molecules, i.e. intermolecular forces are the same as in ice. Indeed the energy of the acoustic wave generated after the collision of the probe with the target particle of the medium, increases from the viscous to the elastic regime, i.e. upon frequency increase, due to the decrease of the acoustic dissipation. The same trend holds for the speed of sound, which manifests a positive dispersion vs frequency, while the opposite is true for the viscosity. Consequently, fingerprints of relaxation phenomena typical of collective dynamics can be found by examining the trend of the liquid physical parameters with frequency [10]. Surprisingly, and this is the demonstration of their ingenious intuitions, this conclusion is exactly the same arrived at by Brillouin and Frenkel in their pioneering works [4,5], that anticipated what has been experimentally assessed about the structure of liquids only a century after. Frenkel in particular introduces the idea that liquids are constituted by a very large number of randomly oriented dynamic pseudo-crystals of submicroscopic size ${ }^{1}$, that continuously rearrange their composition in terms of atoms or molecules. To explain this concept, he uses the relaxation time $\tau_{F}$, introduced for the first time by Maxwell [70], as the average time between particle jumps at one point in space in a liquid [5]; its inverse, $v_{F}=1 / \tau_{F}$, is the frequency of occurrence of the particle jumps. The presence of relaxation time is a distinguishing aspect of liquids vs gases; in the latter every collision is independent from any other, and every collision is equal to the previous and successive ones. The heat motion in liquids close to the crystallization point has the same character as in solids, consisting of oscillations of molecules around their equilibrium positions. The positions of atoms in liquids are of course not permanent but temporary. After performing a number of oscillations around a given position, the atom can jump to another equilibrium position, far $\delta$ from the previous, of the same order of magnitude as the average distance among molecules in that liquid. This step-by-step wandering, lasting a time $\tau_{F}$, constitutes a sort of self diffusion motion, leading to a gradual mixing up of all the atoms [see for instance 53,54]. It must proceed much faster in liquids than in solids, and must have a simpler character because of the absence of definite lattice sites. Figure 2 illustrates the scenario. The relaxation time $\tau_{F}$ has two intrinsic limits [see for instance $11,25,26,34$ ]. The upper limit is at low temperatures, anyway above the crystallization point, to leave the atoms the possibility to jump between two equilibrium positions. This limit corresponds to time intervals $\tau_{F} \approx 10^{2} \div 10^{3} \mathrm{~s}$ typical of the glass transition. The lower limit corresponds, at high temperature, to the minimum value of the Debye vibration period, $\tau_{F}=\tau_{D}=1 / v_{D} \approx 0.1 p s$, and the time interval between two successive jumps, the relaxation time, becomes comparable with the shortest vibration period. Thus the way of distinguishing a liquid from a solid or a gas goes

\footnotetext{
${ }^{1}$ In his book [5] Frenkel actually affirms that this idea was introduced by Stewart around 30's; Stewart proposed to denote such submicroscopic crystals, consisting of few tens of molecules, at most, by the term "cybotactic group" (or regions), and assumed them to be connected with each other by thin layers of the wholly amorphous phase.
} 
around the values of the relaxation time $\tau_{F}$. If, at a given temperature, the characteristic time $t$ of a perturbation propagating in the liquid is much shorter than $\tau_{F}$ (or the frequency $f$ much larger than $v_{F}$ ), over the time interval $t$ the medium is seen by the perturbation as a solid, the particles have no time to rearrange. The dynamic response is that of a free recoil of the liquid particle after the elastic collision with the probe (neutron or X-ray photon). The emerging spectrum is that of a solid being determined by the classical Maxwell-Boltzmann distribution [24], while the speed of sound is much higher. This case is that of "fast sound", whose velocity for water of $\approx 3200 \mathrm{~m} / \mathrm{s}$ obtained experimentally $[13-15,20]$ is very close to the sound velocity found in crystalline ice $\mathrm{I}_{\mathrm{h}}$, of $\approx 4000 \mathrm{~m} / \mathrm{s}$, confirming that, in the explored range of temperature, water is in the viscoelastic regime. In this configuration the medium supports one longitudinal mode and two transversal modes. At time significantly longer than $t$ the opposite is true, the system is seen as a viscous medium and it supports only one longitudinal mode.

What described above is equivalent to assert that liquids and solids differ only quantitatively and not qualitatively [29], the border line being the frequency $v_{F}$, or the relaxation time $\tau_{F}$ (as guessed by Frenkel [5], see also footnote 2). Liquids do not support all vibration modes as solids, but only those above the Frenkel frequency $v_{F}$. More precisely, liquids support 2 transversal and 1 longitudinal modes at low temperature; as far as the temperature increases approaching the liquid-to-gas transition, the capability of supporting shear stresses is lost, and only the longitudinal mode survives. This limit provides the border line between purely diffusive motions and vibratory, or oscillatory, motions. This topic is duly discussed later.

The experimental evidences shortly described above may be summarized as follows:

1. propagation of light in liquids is affected by Doppler shift;

2. specific heat of monatomic liquids exhibits a temperature dependence typical of a substance in which a crystalline structure is present; in particular, it decreases from about $3 R$ at the melting point down to $2 R$ at the critical point;

3. when liquids are kept in controlled isothermal conditions, dynamic collective modes, both longitudinal and transversal, have been detected at very-high-frequencies, i.e. very-short-wavelengths;

4. these modes have been found to propagate in liquids at speeds close to that of ordinary sound in the corresponding solid, correlation lengths being higher than those normally attributed to liquids, which behave as a rigid network on such mesoscopic scale;

5. non-isothermal experiments performed in pure liquids and mixtures far from critical conditions have shown that the symmetry breaking induces very-long-range correlation fluctuations, increasing even more the correlation lengths.

We will show in following sections that the above experimental results may be interpreted within the DML. In this model, liquids are modelled as made of solid-like clusters in dynamic continuous re-arrangement, wandering in a liquid ocean. Clusters, or "liquid particles", interact with a population of elastic wave-packets (throughout this paper we will use the terms wave-packets, phonons, collective excitations, interchangeably), exchanging with them energy and momentum. Let us then proceed to describe this model. 


\section{The Dual Model of Liquids (DML).}

This paragraph is made of four sections. In the first a description is provided of how the wave-packets work as thermal excitations and carriers of energy and momentum in the DML. The expression for the interaction term is introduced. In the second section an expression for the gradient of elastic pressure is derived based on "first principles" of wave mechanics. It is shown that it may be seen as a thermal pressure gradient, giving rise to an inertial term, the same introduced in the previous section, working when the characteristics of the medium change, continuously or abruptly, along the longitudinal wave propagation direction. In the third section the wavepacket $\leftrightarrow$ liquid particle interaction is described, while in the last section the pivotal role of relaxation times is duly analyzed, as well the consequences of the model on the energy associated with the collective modes; their OoM is also calculated and compared with the PLT forecasts.

\subsection{Thermal excitations in liquids.}

The large differences in transport phenomena between solids and gases are due to the different underlying mechanisms of molecular interactions. In gases interactions among molecules are very weak, resulting in random motions and slow diffusion. In solids the molecules are so strongly bounded to the lattice sites that gradient of concentration or moderate gradient of electrical potential cannot dislodge them. A moderate temperature gradient is certainly unable to dislodge molecules from their lattice sites, but solids exhibit elevated thermal conductivities, especially isotropic pure crystals, such as diamond. This behaviour may be explained by the Debye model of solids, in which thermal (and elastic) energy is transported by waves (phonons). In a perfect solid lattice, in which the potential energy is quadratic in the distance from the equilibrium positions, elastic and thermal perturbations are carried by harmonic waves, able as such to transport only energy. Of course heat diffusion in solids, including perfect crystals, is not instantaneous, because the potential energy is not exactly quadratic in the distance between the two scatterers, and anharmonic terms arise. A typical example of such a potential is the Lennard-Jones. This picture may be applied also to the liquid state, where the number of anharmonicities is much larger than in solids, as confirmed by the difference in the Grüneisen constant of various substances in the solid and liquid states, which constitutes a proof of the presence of anharmonicity in liquids and of its relative importance in their behaviour. The fundamental role of anharmonicities consists in make it possible to exchange also momentum other than energy, providing thus an explanation for the diffusion and thermal diffusion in liquids.

The value of $\approx 3200 \mathrm{~m} / \mathrm{s}$ for the speed of sound in liquid water, measured on mesoscopic scale up to distances of several molecular diameters is one of the driving argument of the DML, in which liquids are depicted as constituted by dynamic clusters, solid-like aggregates of molecules of the liquid in continuous re-arrangement, fluctuating and interacting with the liquid global system. Solid consists of only a crystalline, or pseudo-crystalline, phase. As the temperature increases, the fusion is not instantaneous, the solid phase giving progressively way to clusters swimming in an ocean of amorphous liquid. Liquid molecules are bounded to the local lattice of the cluster to which they belong; the number and size of these domains of coherence, i.e. the clusters (or elsewhere dubbed also dynamic icebergs) decrease and the amount of amorphous liquid increases, to the point where it reaches the pure liquid (at the historically-called Frenkel line). As far as thermal (elastic) perturbations propagate within such a cluster, they 
behave as in solids. When perturbations cross the boundary between two such local lattices ${ }^{2}$, inertial effects develop and the interactions are accompanied by propagation of elastic energy in forms of wave-packets because of the anharmonicity of the potential field arising at the cluster' border. Anharmonicity makes the interaction time non negligible and thus allows for momentum transport giving rise to the displacement of the icebergs, i.e. to their diffusion (Figure 1) [39-41,44-45,71]. In this frame the elastic waves travel along the solid-like local lattice at speeds close to that of the corresponding solid phase; when the perturbations cross the boundaries of the local domains, their speed decreases and the other liquid parameters undergo the same fate.

This arrangement of liquid molecules on mesoscopic scale along with local lattices justifies the experimental value found for the speed of sound in water (and in other liquids) close to that of the corresponding solid form [13-15]. This last aspect confirms also the Positive Sound Dispersion observed in liquids with respect to the frequency [10-12] (even if a formal expression for $d u^{w p} / d v$ is not available yet in the DML).

It is clear from above that what we usually mean as a "liquid" is not a liquid at all, but a mixture of solid-like clusters of molecules in continuous re-arrangement and an amorphous, disordered liquid phase. As consequence, any liquid parameter experimentally measured at temperatures and pressures where a usual liquid phase exists, is actually a pondered average of a solid/liquid value.

Let's now translate the ideas illustrated above into a model of the liquid state using the laws of physics. Figure 3 represents the "liquid particle $\leftrightarrow$ wave-packet" interaction, and shows how these interactions work. As outlined above, one of the most important aspects is that, involving wave-packets and not harmonic waves, the interaction with the liquid particle takes a finite time $\left\langle\tau_{p}\right\rangle$, during which the cluster is displaced by $\left\langle\Lambda_{p}\right\rangle$ (here and in the rest of the paper, the two brackets \langle\rangle indicate the average over a statistical ensemble of the quantity inside them). During the interaction, both energy and momentum are exchanged between the phonon and the liquid particle. In an event of type a) an energetic wave-packet collide with a dynamic iceberg, transferring to it the energy $\Delta \varepsilon^{w p}$ and momentum $\Delta p^{w p}$, given by:

$$
\begin{aligned}
& \text { 4. } \Delta \varepsilon^{w p}=h\left\langle v_{1}\right\rangle-h\left\langle v_{2}\right\rangle=\Delta E_{p}^{k}+\Delta \Psi_{p}=f^{\text {th }} \cdot\left\langle\Lambda_{p}\right\rangle \\
& \text { 5. } \Delta p^{w p}=f^{\text {th }} \cdot\left\langle\tau_{p}\right\rangle .
\end{aligned}
$$

In Eq.(4) $\left\langle v_{1}\right\rangle$ and $\left\langle v_{2}\right\rangle$ represent the wave-packet frequency before and after the collision, respectively. $\Delta E_{p}^{k}$ is the kinetic energy acquired by the liquid particle, while $\Delta \Psi_{p}$ is that part of the energy lost by the wavepacket in the inelastic interaction and converted into potential energy of internal collective DoF of the solid-like cluster. Finally $f^{\text {th }}$ is the interaction term between the wave-packet and the liquid particle. The wave-packet emerges from the collision with reduced energy and momentum, while the cluster acquires the energy and momentum lost by the phonon. The effect is of having converted the energy carried by the wave-packet into kinetic and potential energy of the cluster. The power developed during the interaction is:

\footnotetext{
2 In case of solutions, this same behaviour shall be observed among a cluster of solvent and a solute particle; solute particles of course will have a more complicate structure, but however comparable to that of a cluster of pure solvent from the point of view of the heat current propagation.
} 
6. $W=f^{\text {th }} \cdot \frac{\left\langle\Lambda_{p}\right\rangle}{\left\langle\tau_{p}\right\rangle}$.

The kinetic energy will be dissipated as friction against the liquid. The liquid particle will begin relaxing the potential energy $\Delta \Psi_{p}$ once the interaction is completed, i.e. after $\left\langle\tau_{p}\right\rangle$, dissipating it during a time lapse $\left\langle\tau_{R}\right\rangle$, and travelling over a distance $\left\langle\Lambda_{R}\right\rangle$, at the end of which this remaining energy stored into internal DoF is given back to the liquid pool. The total duration of the process and the total displacement of the particle, respectively, are:

$$
\begin{aligned}
& \text { 7. }\langle\tau\rangle=\left\langle\tau_{p}\right\rangle+\left\langle\tau_{R}\right\rangle \\
& \text { 8. }\langle\Lambda\rangle=\left\langle\Lambda_{p}\right\rangle+\left\langle\Lambda_{R}\right\rangle
\end{aligned}
$$

The overall effect of the interaction is that the energy subtracted from the phonon' pool returns into it a time interval $\langle\tau\rangle$ later and a step $\langle\Lambda\rangle$ forward. The event of type b) is just the opposite: an energetic cluster collide with a wave-packet and transfers to it energy and momentum. The wave-packet will emerge from the scattering with increased energy and momentum. The net effect is that of having increased the liquid thermal energy carried by wavepackets at expenses of the kinetic and internal energy of the liquid particle (incidentally, $\langle\tau\rangle$ and $\langle\Lambda\rangle$ introduced in Eqs.(7) and (8), have the same meaning of the parameters $\tau_{F}$ and $\delta$ introduced by Frenkel [5], (see Figure 2)). In equilibrium conditions, events of type a) will alternate with events of type b), to maintain the balance of the two energy pools unaltered. Besides, the macroscopic equilibrium is the consequence of the mesoscopic equilibrium; events like those of Figure 3 will be equally probable along any direction, in such a way to have a null average over time and space. On the contrary, if a symmetry breaking is introduced, as for instance a temperature or a concentration gradient, one type of event will prevail over the other along the direction of the gradient. We will return in the "Discussion" on this elementary scattering process. For the moment it is important to notice that, while the kinetic energy is a continuum, the internal vibrational collective DoF are quantized. This means that to excite them, the energy transfer must follow the rules of quantum theory, i.e. only finite quanta of energy can be exchanged between the internal DoF and the external pool; the only possible exception is that of anharmonic oscillators that can generate a wide spectrum of frequencies in the interaction process, as hypothesized in DML. Besides, as we will see in the Discussion, an exchange of energy among internal (vibrational) and external (translational) DoF is made possible under precise circumstances.

The interactions described in Figure 3 have the following characteristics:

a) Both energy and momentum transport are allowed, the collisions being inelastic.

b) Everyone of the two events a) and b) is commuted into the other by time reversal. At equilibrium, the average energy and momentum exchanged in events of type a) are equal to those exchanged in events of type b); in other words, at equilibrium the two events are statistically equivalent and have the same a priori probability to occur. If a symmetry breaking is imposed to the system, as a temperature or a concentration gradient, one of the two events will have a larger probability to occur with respect to the other.

c) Part of the energy transferred by the wave-packet to the liquid particle increases the energy of internal DoF. This is shown in Figure 3a), where on the left there is a non perturbed liquid particle just before the collision with a 
wave-packet takes place. The impact transfers part of the energy of the wave-packet to the cluster; this energy is partly converted into kinetic energy of the cluster, and partly into potential energy, exciting the internal vibration DoF (centre of the figure). The collision lasts $\left\langle\tau_{p}\right\rangle$ and the liquid particle is displaced by $\left\langle\Lambda_{p}\right\rangle$. Once the collision is completed and the phonon has emerged with reduced energy and momentum, the liquid particle de-excites and releases the excess energy in the time interval $\left\langle\tau_{R}\right\rangle$, moving by $\left\langle\Lambda_{R}\right\rangle$ (right side of the figure). In Figure $3 b$ ) the time-reversed event of a) is represented.

One of the possible effects which one would get by accounting for the energy stored in non-propagating modes will not be dealt with here. It has indeed an impact on the heat propagation, a topic that will be accurately dealt with in a separate paper [72].

\subsection{The Radiant Vector and the elastic radiation pressure.}

Having stated that thermal excitations in liquids consist of elastic wave-packets, we now calculate the pressure generated by the flux of elastic energy travelling in a condensed medium. This elastic radiation pressure generates the interaction term $f^{\text {th }}$ responsible of the wave-packet $\leftrightarrow$ liquid particle interaction introduced in Eqs. (4) and (5).

Energy density $E$ associated with longitudinal mechanical waves consists of a kinetic and a potential part:

$$
\text { 9. } E=\left\langle E_{\text {kin }}\right\rangle+\left\langle E_{\text {pot }}\right\rangle=\rho\left\langle\dot{\xi}^{2}\right\rangle
$$

$\rho$ being fluid density and $\dot{\xi}$ oscillation velocity of the particles. The flux of elastic energy $\vec{J}_{e l}$ is $\left(E \cdot u_{g}\right) \vec{r}$ where $u_{g}$ is group velocity and $\vec{r}$ the unit vector along the direction of propagation. On the other hand, it is also:

$$
\text { 10. } \vec{J}_{e l}=(\Delta \Pi \cdot \dot{\xi}) \vec{r}=\vec{R}
$$

$\Delta \Pi$ being the amplitude of pressure variation. The quantity $\vec{R}$, which we call radiant vector [73-74] represents the instantaneous acoustic power in the beam; it is never negative because $\Delta \Pi$ and $\dot{\xi}$ change sign simultaneously every half period. Dividing Eq. (10) by the phase velocity $u_{\varphi}$, one gets the elastic radiation pressure that manifests in a medium along the elastic wave propagation direction, according to the classic Rayleigh approach. If the characteristics of the medium change, a gradient of elastic pressure rises, $\nabla \Pi_{e l}=\nabla\left(\frac{E \cdot u_{g}}{u_{\varphi}}\right)=\nabla\left(\frac{J_{e l}}{u_{\varphi}}\right)$.

The reader should note that the concept of radiant vector holds for longitudinal waves of any frequency and amplitude. As shown in the previous section, elastic wave-packets are also responsible for propagation of heat waves inside condensed media. What was said for the flux of elastic energy can also be said for the flux of thermal energy, as also pointed out by Joyce [74]. We replace the gradient of elastic pressure with the gradient of thermal radiation pressure, provided that the flux of elastic energy is replaced by that of thermal energy, yielding:

11. $\nabla \Pi_{t h}=\nabla\left(\frac{J_{q}}{u_{\phi}}\right)=-\varphi^{t h}$ 
Eq.(11) contains two supplementary information; first, because thermal energy is carried by elastic wave-packets, the velocity of energy propagations remains the same. Second, the gradient of pressure gives rise to an inertial term, $\varphi^{\text {th }}$, responsible for momentum transport associated to the wave-packets propagation. Eq.(11) is valid when the characteristics of the medium change continuously along the wave-packets propagation direction. The same argument may be used when wave-packets impinge on an "obstacle", as for instance a liquid particle: the radiant vector changes and an acoustic radiation pressure is produced on the boundary. Observing that the inelastic character of the collision allows exchange of momentum, this leads to the appearance of a force, $f^{\text {th }}$, acting on molecular clusters. Consequently, Eq.(11) may be re-written in terms of discrete quantities, yielding the following expression for the thermal radiation pressure:

12. $\Delta \Pi_{t h}=\left[\left(\frac{J_{q}}{u_{\varphi}}\right)_{1}-\left(\frac{J_{q}}{u_{\varphi}}\right)_{2}\right]=\frac{f^{t h}}{\sigma_{p}}$.

Wave-packets thus exchange energy $\Delta \varepsilon=f^{t h} \cdot\left\langle\Lambda_{p}\right\rangle$ (Eq.(4)) and momentum $\Delta p=f^{t h} \cdot\left\langle\tau_{p}\right\rangle$ (Eq.(5)) with liquid particles, $\left\langle\Lambda_{p}\right\rangle$ and $\left\langle\tau_{p}\right\rangle$ having the same meaning as before. In other words, change of local order produces at the mesoscopic scale on the wave-packets the same effect of a boundary, resulting in a change of their radiant vectors.

Expressions analogous to Eq.(12) have been previously derived by many Authors in various different approaches. It derives from the Boltzmann-Ehrenfest' Adiabatic Theorem [75], later generalized by Smith [76] and by Gaeta et al. [77]; in a different approach analogous result was obtained calculating the radiation pressure produced by acoustic waves on an idealized liquid-liquid interface [42].

The third member of Eq.(12) provides the expression for $f^{\text {th }}$ introduced in Eqs.(4) and (5),

13. $f^{\text {th }}=\sigma_{p}\left[\left(\frac{J_{q}}{u_{\varphi}}\right)_{1}-\left(\frac{J_{q}}{u_{\varphi}}\right)_{2}\right]$

$\sigma_{p}$ representing the cross-section of the "obstacle", the solid-like cluster, on the surface of which $\Delta \Pi_{t h}$ develops. A final consideration concerns the algebraic sign of $f^{t h}$, or that of $\Delta \Pi_{t h}$ or $\Delta \Pi_{e l}$ : it will be positive or negative depending on the sign of the quantity in square brackets, i.e. on whether the energy associated with the propagation of elastic waves in the medium " 1 " is larger or smaller than in the medium " 2 ".

In the first part of the next paragraph some concepts introduced by Debye and by Gaeta and co-workers are recalled. On this basis, the DML is built without any reference to external temperature gradient applied to the liquid, the expression for the specific heat valid in all three states of matter is derived, and in the last paragraph of this section the relaxation times are defined and the related OoM are calculated. 


\subsection{Wave-packets $\leftrightarrow$ liquid particle interactions and thermal energy associated with collective} modes in the DML.

The existence in liquids of thermal excitations, consisting of high-frequency wave-packets, capable of interacting with the molecules of the liquid, has many interesting consequences. Let's start by considering the propagation of thermal (elastic) energy in liquids due to wave-packets diffusion. The total internal energy $q_{T}$ per unit of volume of a liquid at temperature $\mathrm{T}$ is:

$$
\text { 14. } q_{T}=\int_{0}^{T} \rho C_{V} d \theta=f[\Theta / T]
$$

where $\rho$ is the medium density, $C_{V}$ the specific heat per unit mass at constant volume, $\Theta$ the Debye temperature of the liquid at temperature T. However only a fraction $q_{T}^{w p}$ of this energy is transported by the wave-packets, given by:

$$
\text { 15. } \quad q_{T}^{w p}=m q_{T}=\mathcal{N}^{w p}\left\langle\varepsilon^{w p}\right\rangle=m^{*} \rho C_{V} T
$$

The parameter $m$ has been introduced because the dynamics described above involves the DoF of the lattice; $m$ accounts for the ratio between the number of collective DoF surviving at temperature T, and the total number of available collective DoF. We will return in the following on the meaning and values of $m$, for now it is relevant to note that $0 \leq m \leq 1$. In Eq.(15) $\mathcal{N}^{w p}$ is the number of wave-packets per unit of volume ${ }^{3}$, and $\left\langle\varepsilon^{w p}\right\rangle$ their average energy. As for the last member of Eq.(15), the quantity $m^{*}=m \frac{\int_{0}^{T} \rho C_{V} d \theta}{\rho C_{V} T}$ depends on the nature of the liquid, and has been introduced for the sake of simplifying the expression.

In the absence of any external perturbation to the system, i.e. for an isolated system, the wave -packets will be randomly and isotropically oriented. With reference to Figure 1 and Figure 3, during their free-flight $\left\langle\Lambda_{w p}\right\rangle$ between two successive interactions with the liquid particle, they may be considered as local microscopic heat currents. Each wave-packet propagates along an average distance $\left\langle\Lambda_{w p}\right\rangle$, during a time interval $\left\langle\tau_{w p}\right\rangle$, which is its average lifetime. Accordingly, the ratio $\left\langle\Lambda_{w p}\right\rangle /\left\langle\tau_{w p}\right\rangle$ defines the wave-packet average propagation velocity between two successive interactions,

16. $u^{w p}=\frac{\left\langle\Lambda_{w p}\right\rangle}{\left\langle\tau_{w p}\right\rangle}=\lambda^{w p} \cdot v^{w p}$.

$3 \mathcal{N}^{w p}$ is the average density of the wave-packets statistical distribution. As such $\mathcal{N}^{w p}$ is represented by a Bose-Einstein distribution function. 
where $v^{w p}$ and $\lambda^{w p}$ are the central frequency and wave-length of the wave-packet ${ }^{4}$. Considering a surface $\mathrm{S}$ arbitrarily oriented within the liquid, at equilibrium an equal number of heat currents will flow through it in opposite directions. Let's assume now that locally every heat current, although isotropically distributed in the liquid, is driven by a virtual temperature gradient $\left\langle\frac{\delta T}{\delta z}\right\rangle$ applied over $\left\langle\Lambda_{w p}\right\rangle$. From Eq.(15), the variation of the energy density within the liquid along the " $+z "$ direction is:

17. $\delta_{+z} q_{T}^{w p}=\frac{1}{6} \delta\left(m q_{T}\right)=\frac{1}{6} \delta\left[\mathcal{N}^{w p}\left\langle\varepsilon^{w p}\right\rangle\right]=\frac{1}{6} \mathcal{N}^{w p}\left\langle\Delta \varepsilon^{w p}\right\rangle=\frac{1}{6} \frac{\partial q_{T}^{w p}}{\partial T}\left\langle\frac{\delta T}{\delta z}\right\rangle\left\langle\Lambda_{w p}\right\rangle$

From the third-to-fourth member of Eq.(17) it has been supposed that the wave-packets distribution function $\mathcal{N}^{w p}$ is not significantly altered along the heat current, and that only their average energy changes. The local virtual temperature gradient $\left\langle\frac{\delta T}{\delta z}\right\rangle$ in last member represents the thermodynamic "generalized" force [with the usual meaning of irreversible thermodynamics, 78] driving the diffusion of thermal excitations along $z$. The factor $1 / 6$ accounts for the isotropy of the phonon current through a liquid at equilibrium. By applying this driving force over the distance $\left\langle\Lambda_{w p}\right\rangle$, a wave-packet diffusion, i.e. the heat current $j^{w p}$, is generated along the " $+z^{\prime \prime}$ direction:

18. $j_{+z}^{w p}=-D^{w p}\left(\frac{\delta q_{T}^{w p}}{\delta z}\right)_{+z}=-\frac{1}{6} u^{w p}\left\langle\Lambda_{w p}\right\rangle \frac{\partial q_{T}^{w p}}{\partial T}\left\langle\frac{\delta T}{\delta z}\right\rangle$

where $D^{w p}=u^{w p}\left\langle\Lambda_{w p}\right\rangle$ is the diffusion coefficient of the wave packets. Therefore, the propagation of an elementary heat current is induced by a virtual temperature gradient $\left\langle\frac{\delta T}{\delta z}\right\rangle$ over the distance $\left\langle\Lambda_{w p}\right\rangle$ lasting the time interval $\left\langle\tau_{w p}\right\rangle$

Eq. (18) for the heat current may also be obtained by calculating the energy flowing in a cylinder per unit area and per unit time, i.e.:

19. $j_{+z}^{w p}=u^{w p} \partial_{+z} q_{T}^{w p}=\frac{1}{6} u^{w p} \mathcal{N}^{w p}\left\langle\Delta \varepsilon^{w p}\right\rangle$.

$q_{T}^{w p}$ in Eq.(15) allows for calculating the phonon specific heat contribution $C_{V}^{w p}$

\footnotetext{
4 Although an elastic perturbation of the liquid lattice should be represented by a wave-packet, whose localization depends on the microscopic structure to which it is associated, here it will be represented, for the sake of mathematical simplification, before and after an interaction, as a monochromatic wave, longitudinally polarized, and hence completely identified by its average wave-length $\lambda^{w p}$ and frequency $v^{w p}$, whose product is equal to $u^{w p}$.
} 
20.

$$
\left\{\begin{aligned}
\rho C_{V}^{w p} & =\frac{\partial q_{T}^{w p}}{\partial T}=\frac{\partial}{\partial T}\left(m q_{T}\right)=q_{T} \frac{d m}{d T}+m \frac{\partial q_{T}}{\partial T}=q_{T} \frac{d m}{d T}+m \rho C_{\mathrm{V}}= \\
& =m \rho C_{\mathrm{V}}\left[\frac{q_{T}}{m \rho C_{\mathrm{V}}} \frac{d m}{d T}+1\right]=m \rho C_{\mathrm{V}}\left[\frac{m^{*}}{m^{2}} \frac{d m}{d T} T+1\right]
\end{aligned}\right.
$$

Let's evaluate now the quantity in square brackets at the last member. Because

21. $\rho C_{V}^{w p}=\frac{\partial q_{T}^{w p}}{\partial T} \geq 0$

and $0 \leq m \leq 1$, this implies that $\left[\frac{m^{*}}{m^{2}} \frac{d m}{d T} T+1\right] \geq 0$. As for the temperature dependence of $m$, we will discuss also in Section 4 how this parameter evolves in liquids from the glass transition up to the Frenkel line, and how it is modelled in the DML. The basis for the reasoning is the experimental evidence of the presence in liquids of transversal modes [9,11,13-18,20-24,25,34-36] actives for the propagation of elastic energy by means of shear waves working as in the solid phase. These modes however persist in the liquid phase as long as solid-like structures survive. Experiments show that the two transversal modes disappear when the system approaches the critical point, where only the longitudinal collective modes survive accounting for the compression and rarefaction waves responsible for hydrodynamic modes propagation. We will return on this point, by now it is important to have in mind that the number of collective DoF defined by $m$ decreases with temperature, so that $\frac{d m}{d T}<0$. Consequently, $\frac{m^{*}}{m^{2}} \frac{d m}{d T} T \geq-1$, and this defines the lower limit for $\frac{m^{*}}{m^{2}} \frac{d m}{d T} T$. As for the upper limit, because of the fact that $\frac{d m}{d T}<0$ and $\left[\frac{m^{*}}{m^{2}} \frac{d m}{d T} T+1\right] \geq 0$, we get for maximum value $\frac{m^{*}}{m^{2}} \frac{d m}{d T} T \leq 0$. Definitively we may conclude that

22. $0 \leq\left[\frac{m^{*}}{m^{2}} \frac{d m}{d T} T+1\right] \leq 1$

and finally

23. $C_{V}^{w p} \leq C_{V}$

as one would have expected.

Besides being an energy density, $q_{T}^{w p}$ represents also the pressure $\Pi^{w p}$ exerted by elastic wave-packets in the medium. Compiling Eq. (12) with Eqs.(17), (18) and (20), along "+ $z$ " we get:

24. $\quad \Pi_{+z}^{w p} \equiv \delta_{+z} q_{T}^{w p}=\frac{1}{6} \delta q_{T}^{w p}=\frac{1}{6} \delta\left(\frac{j^{w p}}{u^{w p}}\right)=\delta_{+z}\left(\frac{j^{w p}}{u^{w p}}\right)=\frac{\partial q_{T}^{w p}}{\partial T}\left\langle\frac{\delta T}{\delta z}\right\rangle_{+z}\left\langle\Lambda_{w p}\right\rangle=\rho C_{V}^{w p}\left\langle\Lambda_{w p}\right\rangle\left\langle\frac{\delta T}{\delta z}\right\rangle_{+z}$ 
Let us now return to Eq.(18), which represents the flux of thermal energy carried by a wave-packet propagating over the distance $\left\langle\Lambda_{w p}\right\rangle$ and driven by the virtual gradient $\left\langle\frac{\delta T}{\delta z}\right\rangle$. As such $j_{+z}^{w p}$ may be re-written introducing the (mesoscopic) thermal conductivity of the medium $K^{w p}$ :

$$
\text { 25. } j_{+z}^{w p}=-K_{+z}^{w p}\left\langle\frac{\delta T}{\delta z}\right\rangle_{+z}=-\frac{1}{6}\left\{u^{w p}\left\langle\Lambda_{w p}\right\rangle \rho C_{V}^{w p}\right\rangle\left\langle\frac{\delta T}{\delta z}\right\rangle=-\frac{1}{6} D^{w p} \rho C_{V}^{w p}\left\langle\frac{\delta T}{\delta z}\right\rangle
$$

from which we get:

$$
\text { 26. } K_{+z}^{w p}=\frac{1}{6} D^{w p} \rho C_{V}^{w p}=\frac{1}{6} u^{w p}\left\langle\Lambda_{w p}\right\rangle \frac{\partial}{\partial T}\left[\mathcal{N}^{w p}\left\langle\varepsilon^{w p}\right\rangle\right]=\frac{1}{6} u^{w p}\left\langle\Lambda_{w p}\right\rangle m \rho C_{V}\left[\frac{m^{*}}{m^{2}} \frac{d m}{d T} T+1\right]
$$

What stated so far for the direction " $+z$ " can be analogously stated for the opposite direction " $-z$ ", getting the same expression as Eq.(26) but with the opposite sign; by summing them algebraically, we eventually obtain:

27. $K^{w p}=\frac{1}{3} u^{w p}\left\langle\Lambda_{w p}\right\rangle \frac{\partial}{\partial T}\left[\mathcal{N}^{w p}\left\langle\varepsilon^{w p}\right\rangle\right]=\frac{1}{3} u^{w p}\left\langle\Lambda_{w p}\right\rangle m \rho C_{V}\left[\frac{m^{*}}{m^{2}} \frac{d m}{d T} T+1\right]=\frac{1}{3} u^{w p}\left\langle\Lambda_{w p}\right\rangle \rho C_{V}^{w p}$

Interestingly, Eq. (27) for the (phononic) thermal conductivity has exactly the same form as that derived by Boltzmann for the thermal conductivity due to the particle collision and drift in a fluid medium [79, Eq.(86)]. In particular, the quantity $G$ introduced by Boltzmann is here replaced by $\rho C_{V}^{w p}$, that in turn depends upon the number of DoF pertaining to the particles involved in the process [79, Eq.(88)].

So far the physical implications on the dynamics of pure liquids at equilibrium (the local temperature gradient $\left\langle\frac{\delta T}{\delta z}\right\rangle$

is indeed a virtual quantity accounting for the wave-packets current) deriving from the DML. The main conclusions arrived at are the following. In the DML $f^{\text {th }}$ develops when the characteristics of the medium change; this happens in pure liquids at equilibrium at the boundary of the local ordered domains (i.e. the liquid particles, also dubbed here the dynamic clusters, or again the dynamic icebergs) within which liquids may be considered as solids as long as these domains of coherence last. When the wave-packet hits a liquid particle, the force given by Eq.(13) develops, it acts for $\left\langle\tau_{p}\right\rangle$ displacing the particle by $\left\langle\Lambda_{p}\right\rangle$. Energy $\left\langle\Delta \varepsilon^{w p}\right\rangle$ and momentum $\left\langle\Delta p^{w p}\right\rangle$, given by Eqs.(4) and (5) respectively, are transferred to the particle during $\left\langle\tau_{p}\right\rangle$, increasing its kinetic and potential energy and exciting internal vibratory energy levels. These DoF oscillate similarly to those pertaining to solid state, giving origin to (quasi) elastic waves with characteristic length- and time-scales $\left\langle\Lambda_{0}\right\rangle$ and $\left\langle\tau_{0}\right\rangle$ respectively. $\left\langle\tau_{R}\right\rangle$ later and $\left\langle\Lambda_{R}\right\rangle$ forward they are given back to the wave-packet pool, and the process is repeated a time interval $\left\langle\tau_{w p}\right\rangle$ later and a step $\left\langle\Lambda_{w p}\right\rangle$ forward. The statistical equilibrium of events of types a) and b) of Figure 3 ensures that no macroscopic effects are developed. When a symmetry breaking is imposed to the system, for instance a temperature or a concentration gradient, there will be no more statistical equilibrium of the events of Figure 3 , and an excess of elastic wave-packets is produced according to the direction of the external applied (generalized) force. The local heat currents 
will generate forces and observable effects of these microscopic events will appear in the form of mass and/or energy fluxes. However, the evaluation of the dynamical effects from Eq.(13) in a traditional liquid is not a simple exercise because a "pure liquid phase" exists only at temperatures close to the so-called Frenkel line. In Eq.(13), or Eq.(12), it is therefore difficult to identify which values to use if one wishes to evaluate it in a pure liquid or in a solution. Aware of making a mistake, an evaluation of OoM can be made by assuming, in a pure liquid, for the clusters the values of the solid and for the amorphous phase those of the liquid.

\subsection{Relaxation times Order-of-Magnitude evaluation in the DML and comparison with PLT and with experimental forecasts for visco-elastic transition regions.}

Let us then now explain the concept and meaning of the relaxation time $\langle\tau\rangle$ that one should consider in the frame of DML to account for the delay in the energy (or mass) diffusion. To do this we shall refer to Figure 4 that represents a close-up picture of the "liquid particle $\leftrightarrow$ wave-packet" interaction described in Figure 3a. Being wavepackets and not harmonic waves, the inelastic interaction with the liquid particle takes a finite time $\left\langle\tau_{p}\right\rangle$, during which the particle is displaced by $\left\langle\Lambda_{p}\right\rangle$. Through the interaction both energy and momentum are exchanged between the phonon and the particle. In an event of type a) an energetic wave-packet collide with a liquid particle, or iceberg, transferring to it energy $\Delta \varepsilon^{w p}$ and momentum $\Delta p^{w p}$, given by Eqs. (4) and (5) respectively. $\Delta E_{p}^{k}$ in Eq. (4) is the kinetic energy acquired by the liquid particle as consequence of the interaction, and given by:

28. $\Delta E_{p}^{k}=\frac{1}{2} m_{p}\left(\frac{\left\langle\Lambda_{p}\right\rangle}{\left\langle\tau_{p}\right\rangle}\right)^{2}=\frac{1}{2} m_{p} v_{p}^{2}$

where $m_{p}$ is the mass of the displaced liquid particle.

The particle will begin relaxing the potential energy $\Delta \Psi_{p}$ once the interaction is completed, i.e. after $\left\langle\tau_{p}\right\rangle$, dissipating it during a time lapse $\left\langle\tau_{R}\right\rangle$, and travelling over a distance $\left\langle\Lambda_{R}\right\rangle$, at the end of which the energy stored into internal DoF is given back to the liquid pool. The total duration of the process and the total displacement of the particle are given by Eqs. (7) and (8), respectively ${ }^{5}$. Because we are considering a wave-packet, it is not an infinite wave train, but a localized perturbation, whose extension $\left\langle\Lambda_{w p}\right\rangle$ we may write as a multiple of $\lambda^{w p}$ [see Eq. (16)]:

29. $\left\langle\Lambda_{w p}\right\rangle=n \lambda^{w p}, n>1$.

With reference to Figure 4 , let $\left\langle d_{p}\right\rangle$ indicate the cross extension of the liquid particle. The interaction starts when the phonon collide with the particle, and ends when the phonon leaves the particle, after having crossed it at the speed

\footnotetext{
${ }^{5}$ Actually the time interval between two successive interactions, $\tau_{F}$, may be larger than $\langle\tau\rangle$ because another time interval could elapse after the particle is stopped or has dissipated the internal energy acquired during the interaction. On the other side, the occurrence of multiple interactions can be excluded, as discussed below (see Eq.(40)).
} 
30. $u^{0}=\frac{\left\langle\Lambda_{0}\right\rangle}{\left\langle\tau_{0}\right\rangle}$.

During the interaction, lasting $\left\langle\tau_{p}\right\rangle$, the wave-packet transfers to the liquid particle a non-negligible part of its total energy, $\phi=\frac{h\left\langle v_{1}\right\rangle-h\left\langle v_{2}\right\rangle}{h\left\langle v_{1}\right\rangle}$. A fraction $\alpha$ of this becomes kinetic energy of the iceberg, while the remaining fraction $1-\alpha$ becomes potential energy $\Delta \Psi_{p}$ of internal DoF (quantized vibrational DoF). We may write:

31. $\Delta E_{p}^{k}=\alpha \cdot h\left\langle v^{w p}\right\rangle=\frac{1}{2} m_{p}\left\langle v_{p}^{2}\right\rangle \Rightarrow\left\langle v_{p}\right\rangle=\sqrt{\frac{2 \alpha h\left\langle v^{w p}\right\rangle}{m_{p}}}$

From Figure 4, the total path of the phonon, $\left\langle\Delta^{p h}\right\rangle$, is:

32. $\left\langle\Delta^{p h}\right\rangle=\left\langle\Lambda_{w p}\right\rangle+\left\langle d_{p}\right\rangle+\left\langle\Lambda_{p}\right\rangle+\left\langle\Lambda_{w p}^{\prime}\right\rangle$

where the quantity $\left\langle\Lambda_{w p}^{\prime}\right\rangle$ is the extension of the wave-packet emerging after the interaction, with (central) frequency $\left\langle v_{2}\right\rangle$. Of course the total duration of the interaction experienced by the particle is the same as that experienced by the phonon, but because they travel at different speeds, the covered paths will be different. For the only sake of distinguishing among them, we indicate with $\left\langle\tau_{p}\right\rangle$ the duration of the interaction for the particle, and with $\left\langle\tau^{p h}\right\rangle$ that for the phonon, with

$$
\text { 33. }\left\langle\tau_{p}\right\rangle \equiv\left\langle\tau^{p h}\right\rangle \text {. }
$$

We therefore get:

$$
\begin{aligned}
& \text { 34. }\left\langle\tau_{p}\right\rangle=\frac{\left\langle\Lambda_{p}\right\rangle}{\left\langle v_{p}\right\rangle} \\
& \text { 35. }\left\langle\tau^{p h}\right\rangle=\frac{\left\langle\Lambda_{w p}\right\rangle}{u^{w p}}+\frac{d_{p}}{u^{0}}+\frac{\left\langle\Lambda_{p}\right\rangle}{u^{0}}+\frac{\left\langle\Lambda_{w p}^{\prime}\right\rangle}{u^{w p}}
\end{aligned}
$$

In Eq.(35) at the second member the first term is the time taken by the wave-packet to enter the liquid particle, the second is the time taken by the phonon to cross the liquid particle, the third is the time taken to cover the entire path of interaction, and finally the fourth term is the time taken by the wave-packet to leave the liquid particle.

Two approximations are in order for Eq.(35). First, because the emerging wave-packet has a reduced energy with respect to the colliding one, the wavelength of the former will certainly be larger than the latter; we will use however for it the same wavelength as the colliding one, $\left\langle\Lambda_{w p}\right\rangle$. The second approximation concerns the speed at which the colliding and emerging wave-packets enter and leave the liquid particle. We will use the same speed for both, and equal to the speed of elastic waves inside the liquid particle, $u^{0}$, for which, as described in Section 2 , the value is known from experiments. Ultimately, Eq.(35) becomes:

36. $\left\langle\tau^{p h}\right\rangle \cong \frac{2\left\langle\Lambda_{w p}\right\rangle+d_{p}+\left\langle\Lambda_{p}\right\rangle}{u^{0}}$ 
From Eqs.(32) through (36) we have:

37. $\frac{\left\langle\Delta^{p h}\right\rangle}{\left\langle\Lambda_{p}\right\rangle} \cong \frac{2\left\langle\Lambda_{w p}\right\rangle+\left\langle d_{p}\right\rangle+\left\langle\Lambda_{p}\right\rangle}{\left\langle\Lambda_{p}\right\rangle}=\frac{2\left\langle\Lambda_{w p}\right\rangle+\left\langle d_{p}\right\rangle}{\left\langle\Lambda_{p}\right\rangle}+1=\frac{u^{0}}{\left\langle v_{p}\right\rangle}$

from which, considering the last two members, one gets

38. $\frac{2\left\langle\Lambda_{w p}\right\rangle+\left\langle d_{p}\right\rangle}{\left\langle\Lambda_{p}\right\rangle}=\frac{u^{0}}{\left\langle v_{p}\right\rangle}-1$

and finally

39. $\left\langle\Lambda_{p}\right\rangle=\frac{\left\langle v_{p}\right\rangle\left(2\left\langle\Lambda_{w p}\right\rangle+\left\langle d_{p}\right\rangle\right)}{u^{0}-\left\langle v_{p}\right\rangle}$

Compiling Eqs.(31), (35) and (39) it is now possible to get $\left\langle\tau_{p}\right\rangle$ (or $\left\langle\tau^{p h}\right\rangle$ ).

It is easy to evaluate also the time $\left\langle\tau_{\eta}\right\rangle$ needed for a liquid particle to return at rest after the interaction, by supposing that the kinetic energy acquired is dissipated against friction in the liquid, $\Delta E_{p}^{k}=E_{p}^{\eta}$. Although $\left\langle\tau_{\eta}\right\rangle$ has apparently nothing to do with $\left\langle\tau_{R}\right\rangle$, it can tell us after how long time the particle stops and how much space it will have travelled before a successive interaction, provided that no other collisions will have occurred during its flight. To answer such doubt we may get help evaluating the Order-of-Magnitude (OoM) of the phonon density $\mathcal{N}^{w p}$ that can be deduced from Eq.(14); it provides the variation of the energy density $\delta q_{T}^{w p}=\delta\left(m q_{T}\right)$ within the liquid along a given direction. This energy variation is equal to the energy shift associated to a Brillouin doublet [see Eq.(1)] multiplied by the phonon density, $\mathcal{N}^{w p}\left\langle\Delta \varepsilon^{w p}\right\rangle$. Definitively, in water, with a value of 1 calorie for the specific heat and with the same values for the wave-packet frequency as those given above, one has:

40. $\mathcal{N}^{w p}\left\langle\Delta \varepsilon^{w p}\right\rangle=\mathcal{N}^{w p} h \Delta\left\langle v^{w p}\right\rangle=\delta\left(m q_{T}\right)=m \rho C_{V} \Delta T \Rightarrow \mathcal{N}^{w p}=\frac{m \rho C_{V} \Delta T}{\left\langle\Delta \varepsilon^{w p}\right\rangle} \approx m \cdot 4 \cdot 10^{22} \mathrm{~cm}^{-3}$

The OoM of the density of phonons, dealt with as lattice particles, in Eq.(40) is then of the same OoM as the molecular density (for water the molecular density holds $\approx 4 \cdot 10^{22} \mathrm{~cm}^{-3}$ at ambient temperature), or even lesser, because $0 \leq m \leq 1$. This result supports the hypothesis of neglecting multiple interactions of wave-packets with particles. The numerical result of Eq.(40) is obtained assuming that the density of phonons do not change by changing the temperature, the only variation being that of their energy. Therefore the above considerations tell us that $\left\langle\tau_{\eta}\right\rangle$ represents a lower limit for the remaining time interval after $\left\langle\tau_{p}\right\rangle$ before another interaction with a phonon takes place (a liquid particle however may interact with a phonon or may dissipate its internal excess energy also some time after it has stopped). A dedicated analysis of the variation of $\mathcal{N}^{w p}$ with temperature or frequency in the object of a separate paper.

Using the Stokes-Einstein expression, the energy balance equation is:

$$
\text { 41. } \frac{1}{2} m_{p}\left\langle v_{p}^{2}\right\rangle=6 \pi \eta_{l}\left\langle r_{p}\right\rangle\left\langle v_{p}\right\rangle \Rightarrow\left\langle v_{p}\right\rangle=\frac{d x_{p}}{d t}=\frac{12 \pi \eta_{l}\left\langle r_{p}\right\rangle}{m_{p}} x_{p}
$$


where $\left\langle r_{p}\right\rangle=\left\langle d_{p}\right\rangle / 2$, from which we easily get:

42. $\frac{d x_{p}}{x_{p}}=\frac{d t}{\left\langle\tau_{\eta}\right\rangle}$ with $\left\langle\tau_{\eta}\right\rangle=\frac{m_{p}}{12 \pi \eta_{l}\left\langle r_{p}\right\rangle}$

The distance travelled by the liquid particle is obtained by integrating Eq. (42), getting:

$$
\text { 43. } x_{p}(t)=\left\langle v_{p}\right\rangle\left\langle\tau_{\eta}\right\rangle\left(1-e^{-t /\left\langle\tau_{\eta}\right\rangle}\right) \text {. }
$$

To provide a numerical estimation of the OoM of the several variables introduced above, we suppose to be in water at $T=300 \mathrm{~K}$ as example. We have considered several ranges for all the parameters; this has allowed us to evaluate the minimum and maximum values of the variables characterizing the dynamics of wave-packets and liquid particles. In doing this we have also taken into account the fact that, for a wave to "see" an obstacle and interact with it, its wavelength must be less than, or at most comparable with, the size of the obstacle: $\left\langle\Lambda_{w p}\right\rangle \leq\left\langle d_{p}\right\rangle$. Results are provided in Table 1, from which several conclusions may be deduced. First, although $\left\langle\tau_{\eta}\right\rangle$ represents at the best only an OoM for $\left\langle\tau_{R}\right\rangle$, it is clear that the time interval that dominates the dynamics is $\left\langle\tau_{p}\right\rangle$, by far much larger than $\left\langle\tau_{\eta}\right\rangle \cdot\left\langle\tau_{R}\right\rangle$ is the average time taken by the internal (quantized) DoF of the iceberg to de-excite after a collision of the iceberg with a wave-packet has taken place. At present we have no tools to evaluate $\left\langle\tau_{R}\right\rangle$, being them however out of the physical context around which the DML is developed. A detailed quantum mechanical modelling of the internal DoF is the best suitable candidate able to evaluate $\left\langle\tau_{R}\right\rangle$. Anyway, we may certainly conclude from Table 1 that the OoM of total relaxation time is $\langle\tau\rangle\rangle\left\langle\tau_{p}\right\rangle \approx 4 \div 13 \cdot 10^{-12} s$, i.e. of the order of several ps as theoretically foreseen and experimentally deduced from scattering spectra.

Very interestingly, multiplying these values by the corresponding phonon frequencies used in the calculations, $v^{w p} \approx 0.9 \div 3 \cdot 10^{12} \mathrm{~Hz}$, we get:

$$
\text { 44. } v^{w p} \cdot \tau \approx 3.6 \div 40 \text {. }
$$

This result is in line with what foreseen from the PLT and deduced from experimental basis, thus confirming that in the considered ranges of temperature and pressure, the liquid is in a full visco-elastic regime, with a prominence towards the single particle, elastic regime at higher frequencies. This in turn is the evidence of the presence of pseudocrystalline, solid-like structures that manifest through the enhancement of the sound velocity, as well as of anharmonic interactions arising at the border of such structures.

\section{Discussion.}

Aim of this work is to illustrate the physical model able to interpret the experimental phenomenology of liquid dynamics. The proposed DML assumes that liquid molecules are arranged at mesoscopic level on solid-like local lattices in continuous re-arrangement. Within these local domains of coherence, propagation of perturbations occurs at characteristic timescales typical of solids, while the local clusters may interact each other by means of inelastic wave- 
packets exchanging with them energy and momentum. The interaction mechanism proposed is symmetric upon time reversal and the interaction term $f^{\text {th }}$ is deduced on the basis of "first principles".

The OoM calculation of the relaxation times, in particular of $\left\langle\tau_{p}\right\rangle$, is made possible by looking intimately on how a lattice particle, i.e. a wave packet, and a liquid particle, i.e. a dynamic iceberg, interact, as shown in Figure 3 and Figure 4. Calculations based on a kinetic approach show that in the case of water at ambient conditions the liquid particle is in full visco-elastic regime, with a prominence to the single particle, elastic regime by increasing the exciting frequency, as foreseen in the PLT and evidenced by experiments. In this conditions the particle may exchange energy and momentum with all the 3 types of DoF, 2 transversal and 1 longitudinal. This is due to the presence of solid-like structures in which energy propagates at the speed $\left\langle u^{0}\right\rangle$ as in solids, i.e. with reduced dispersion (harmonic waves). When the perturbation reaches or leaves a solid-like structure, anharmonic interactions arise travelling at the speed $\left\langle u^{w p}\right\rangle$, giving origin to a cut-off in the speed of sound and determining the transport of energy and momentum, allowing the structure to displace inside the liquid, alike a self-diffusion motion. This arrangement of liquids justifies the experimental values found on mesoscopic scale for the speed of sound in liquids close to that of the corresponding solid form, and also accounts for the Positive Sound Dispersion found at $\mathrm{THz}$ frequencies. When the speed of sound is measured at small wavelengths, i.e. on distances comparable to the extension of a local domain, a solid-like value $\left\langle u^{0}\right\rangle$ is measured. By increasing the distance over which the sound speed is measured, hence on hydrodynamic scale, the classical value is found.

At equilibrium the flux of wave-packets is driven by the difference of elastic radiation pressure (Eq.(11) or (12)), that manifests through a local virtual temperature gradient $\left\langle\frac{\delta T}{\delta z}\right\rangle$ having the role of a generalized thermodynamic force. This is in some way equivalent to assuming a Local Thermodynamic Non Equilibrium determined by the heat current, although isotropically distributed in the liquid. The Non Equilibrium character is however related only over distances of the order of the wave-packet mean free path $\left\langle\Lambda_{w p}\right\rangle$. When the symmetry of the system is broken, for instance by imposing a temperature or a concentration gradient, the correlation lengths increase up to some millimetres, according to the intensity and orientation of the gradients [60-62] (and to other experimental constraints). In the DML the presence of a gradient induces a preferential direction in the orientation and propagation of local, virtual heat currents (the flux of wave-packets). This flux is responsible for mass and energy transport in liquids out of equilibrium. The elementary scattering events shown in Figure 3 also cope with the presence of the two symmetric Brillouin lines in scattering experiments performed in liquids. As is well known [see for instance 3,50-52,65,80], the Brillouin spectrum in liquids is due to the interaction of the photons of the impinging light beam with the liquid' phonons constituting the heat current. The central line is at the same frequency of the impinging beam and hence collects the energy due to elastic, or quasi elastic, scattering. The two Brillouin lines are instead shifted by a frequency proportional to the speed of sound in the liquid. This means that Brillouin lines account for inelastic scattering. At equilibrium, the two Brillouin lines are perfectly symmetric, because the amount of energy removed from the phonon' pool (Stokes line) by the impinging light beam is the same given back to the phonon' pool (anti-Stokes line). This is justified in the DML by the fact that the two events a) and b) of Figure 3 are equally likely to occur (microscopic time reversibility). This last point allows to recall the attention of the reader on a very interesting aspect related to this 
elementary interaction mechanism at the base of the DML. The symmetry of this interaction upon time reversal should be related to the Onsager reciprocity relations [81-82], that require the existence of linear universal laws for molecular fluctuations to justify the time reversibility. It is proposed here that the elementary interaction mechanism shown in Figure 3 and the non equilibrium statistics of phonon-particle interactions represent the physical base of the Onsager Principle. Incidentally, this was already pointed out in [44] where the heats of transport were calculated for a non isothermal liquid without making use of the Onsager Principle, and getting an expression identical to that obtained in the frame of Irreversible Thermodynamics [78].

When a temperature gradient is imposed to the liquid, the two Brillouin lines do not have anymore the same intensity [60-62,65, see also Section 2 and Eqs. (1-3)]. To an external temperature gradient $\nabla T$ corresponds a heat flux (in the opposite direction), and therefore an increase of "wave-packet $\leftrightarrow$ liquid particle" collisions at a given $\hat{q}$ vector; the intensity of the line accounting for this increase along the direction of the heat flux will be greater than the symmetric one by an amount proportional to $1 / q^{2}$ (see Eq. (3)). In this case, events of type a) have a larger probability to occur than events of type b). Of course, if the symmetry is broken by a particle concentration gradient instead of a temperature gradient, the events having a larger probability of occurrence will be the b). To quantify such reasoning, let's assume $\frac{d T}{d z}=\frac{T_{1}-T_{2}}{L}$ be the temperature gradient externally applied to a system. As before, we assume here that the application of an external temperature gradient has the only effect of changing the average energy $\left\langle\varepsilon^{w p}\right\rangle$ of the wave-packets, their total number $\mathcal{N}^{w p}$ remaining unchanged. Let $\left\langle v_{p}\right\rangle$ be the average number per second of "wave-packet $\leftrightarrow$ liquid particle" collisions at $T_{0} \equiv \frac{T_{2}+T_{1}}{2}$ in the isothermal liquid; due to the presence of the virtual temperature gradient, which is equally present in all the three directions, for each direction this number becomes $\left\langle v_{p}\right\rangle / 6$. If an external (small) temperature gradient $\frac{d T}{d z}$ is applied to the liquid, the total number of collisions per second $\left\langle v_{p}\right\rangle / 6$ may be assumed to stay constant because we have supposed that the number of wavepackets does not change, but for collisions occurring in the direction of heat propagation there will be $\delta\left\langle v_{p}\right\rangle$ excess of collisions per second, and an equal defect $-\delta\left\langle v_{p}\right\rangle$ in the opposite direction. If the intensity of the temperature gradient is not too much high, it is reasonable to assume that there will be no non-linear effects and that the initial state of the system at the microscopic level locally continues to be similar to the one at uniform temperature, except for thermal excitations along $\mathrm{z}$. This means that a liquid particle at a given place in the liquid, where the temperature is $T_{0}$, experiences the same number of collisions per second as if the temperature was uniform; the only difference with the isothermal case is that there is an imbalance in the number of collisions with wave-packets that are originated in the two half-spaces along $z$, one hotter and the other cooler. In the linear range we may assume that $2 \cdot \delta\left\langle v_{p}\right\rangle$ is proportional to the temperature gradient $\frac{d T}{d z}$ externally applied to the liquid. Consequently, the situation will consist 
of a heat flux $J_{q}^{e x t}=-K_{l} \frac{d T}{d z}$ superimposed to two equal and opposite heat fluxes $+j_{+z}^{w p}=-K^{w p}\left\langle\frac{\delta T}{\delta z}\right\rangle_{+}$and $j_{-z}^{w p}=-K^{w p}\left\langle\frac{\delta T}{\delta z}\right\rangle$. Thus obviously:

45. $\frac{2 \cdot\left\langle\delta v_{p}\right\rangle}{2 \cdot\left\langle v_{p}\right\rangle / 6}=\frac{d T / d z}{\langle\delta T / \delta z\rangle}$.

The rationale of Eq. (45) is that macroscopic and microscopic (actually mesoscopic) heat conduction obey the same phenomenological relations with identical coefficients, as Onsager pointed out in his famous papers [see for instance 81-82]. Accordingly, an external temperature gradient $\frac{d T}{d z}$ increases by $\frac{d T / d z}{\langle\delta T / \delta z\rangle}$ the flux of phonons with respect to that corresponding to the local microscopic heat currents $j^{w p}$ due to spontaneous phonon diffusivity. Eq.(45) thus gives the ratio of the number of "wave-packet $\leftrightarrow$ liquid particle" collisions per second due to the applied gradient, to that due to the random motions of collective thermal excitations. The clusters therefore will experience $2 \delta\left\langle v_{p}\right\rangle$ collisions in excess along the direction of the heat flux and will execute as many jumps per second of average length $\langle\Lambda\rangle$ in excess in the same direction. Consequently, every particle travels the distance $2\langle\Lambda\rangle \delta\left\langle v_{p}\right\rangle$ every second along the direction of heat flux generated by the external temperature gradient. This quantity represents the drift velocity $\left\langle v_{p}^{\text {th }}\right\rangle$ of the molecule along $\mathrm{z}$ due to the external temperature gradient:

46. $\left\langle v_{p}^{\text {th }}\right\rangle=2\langle\Lambda\rangle\left\langle\delta v_{p}\right\rangle=\frac{\langle\Lambda\rangle\left\langle v_{p}\right\rangle}{3} \frac{d T / d z}{\langle\delta T / \delta z\rangle}$

The above argument can be applied in principle, and with the obvious adaptations, also to solute particles dissolved in liquid solution and subject to a temperature gradient.

Let's now come to Eq.(20), that deserves a dedicated analysis because it has many interesting consequences. First of all, it should be compared with the expression given by PLT model [7,25-34] for the same physical quantity. The Authors calculate an expression for the harmonic and anharmonic contributions to the specific heat within a liquid phonon theory based on the concept of liquid relaxation time introduced by Maxwell [70]. This approach [7], confirmed by experiments in 21 different liquids, covers the cases of gaseous, liquid and solid states of matter, giving for $C_{V}$ a theoretical limit value of $3 \Re$ for solids down to $2 \Re$ for liquids. Eq. (20) does not allow in the present form an exact evaluation of its dependence upon temperature (or other parameters). Anyway, we want to highlight that the value of the phononic contribution provided by Eq.(20) is always less than that of the total specific heat, as indicated in Eq.(23), the equality being valid in our hypothesis at low temperatures (at the glass transition), where $m \approx 1$ and $\frac{d m}{d T} \approx 0$, and the phonons contribute about $100 \%$ to the total specific heat. On the contrary, the phononic contribution disappears at high temperatures, where there are no more collective excitations in the liquid, and the total specific heat thus decreases to lower values. The above behaviour is reflected by the variation vs temperature of $m$, 
$\frac{d m}{d T}<0$. Accurate and interesting analysis and comparison of the two expressions for the isochoric specific heat are the topics of a separate paper, presently in preparation [83].

Connected with the above argument is the recent theoretical findings along with phonons may carry gravitational negative mass [84]. In their paper, Esposito and co-workers have shown that sound waves, constituted by phonons, not only transport mass during their propagation through condensed media (in the DML phonons carry also momentum), but also that such associated mass is negative. Indeed, in a density gradient they move towards a medium with a lower density, where the impedance is higher and their speed is lower. Of course this theoretical result could have a huge impact on what said before about the phononic contribution to the total heat content of a liquid (Eq.(15) through Eq.(21) and Eq.(23)). If on one side Eq. (23) remains valid in principle, on the other side the phononic contribution to the total pool of thermal (and elastic) energy makes the "classical" part even more prominent with respect to the (negative) part due to collective modes. Indeed, the phononic heat content provided by Eq. (20) turns out to be negative, because the inequality in Eq. (21) is no more valid (and consequently also that in Eq. (22)). It is then clear that, if the DML described above, the considerations made by all many Authors about the experiments performed on liquid systems by means of Brillouin scattering and the theoretical forecasts on the negative mass contributed by collective excitations are all valid arguments, an important link is still missing between theory and experiments on liquid structure, at least as it concerns the interpretation of the negative mass contributed by phonons in the experimental results. A first elementary, though puzzling, question to be answered is whether the II Principle is valid for a system in thermal equilibrium, in which phonons may subtract heat to the pool when colliding with the liquid particle, just because of the presence of an external gravitational field, responsible for the establishment of a density gradient in the isothermal liquid. In other words, is the Clausius postulate applicable to an isothermal system in presence of a gravitational field? Is the presence of this external field enough to allow phonons to subtract heat to an isothermal system? And again, why do phonons prefer the more difficult path than the easier one? What makes easier the path that is normally the most difficult?

Some considerations are in order also for Eq.(27). First, as for $C_{V}^{w p}, K^{w p}$ accounts only for the fraction of thermal energy transported by collective thermal (elastic) excitations of the liquid crystalline lattice driven by the local virtual temperature gradient $\left\langle\frac{\delta T}{\delta z}\right\rangle$. Whether this is a small or a large fraction of the total, or accounts for the whole thermal energy, depends on various circumstances. In a crystalline solid this fraction should represent the total heat transported; indeed for such media, thermal (and elastic) energy is carried by means of phonons. In a metallic solid this is no longer true, due to the contribution of free electrons. What is the situation in ordinary liquids is not yet perfectly clear. According to the model presented here, the relative importance of $K^{w p}$ with respect to $K_{l}$ will depend, besides its direct evaluation, on the fraction (given by $m$ in Eq.15) of heat transported by collective excitations. As it concerns the dependence on temperature of $K^{w p}$ for liquids, it is mainly due to that of $u^{w p}$, and hence to that of $\left\langle\Lambda_{w p}\right\rangle,\left\langle\tau_{w p}\right\rangle$ and to a minor extent to that of $\frac{d m}{d T}$. Evaluation of these quantities in some liquids may allow the calculation of $K^{w p}$ and of the involvement of collective oscillations in carrying heat in liquids. With the exception of water, that shows a behaviour strongly influenced by the hydrogen bonds, thermal conductivity of common liquids decreases with temperature, as expected also in the DML frame; this is at least due to the presence of the quantity in 
square brackets, that decreases with temperature (as also the other quantities in the Eq.(26) for $K^{w p}$ ). From the DML point of view, the decrease of $K^{w p}$ with temperature is due to the decrease of the number and size of the solid-like dynamic icebergs, as pointed out before. The presence of the terms in square brackets, in turn, ensures the positive definition of $K^{w p}$ through Eq.(20). Nevertheless, the considerations raised for Eq.(20) related to the negative contribution of the phononic part to the total pool of energy content of a liquid, are valid even for Eq.(26).

A final consideration on $K^{w p}$ is that the DML opens to a heat propagation in liquids modelled in terms of a Cattaneo equation. Indeed the delay-time requested by this kind of equation is physically justified, representing the average time spent by heat in non-propagating forms. Interestingly, it has been shown [85] that in a thermo-elastic model for liquids, in which Eq.(13) is assumed as a constitutive equation for the system, the consistency of the model with the II Principle is ensured only if a Cattaneo equation is assumed to hold for heat propagation. This argument is the subject of a separate paper [72].

Some remarks on the role of relaxing phenomena acting in such a system are also in order. We have to return to Eqs.(4) and (5), where the energy and momentum exchanged between solid-like clusters of liquid molecules and wavepackets have been defined, and to Eqs.(7) and (8), where the time lapses and particle displacements involved in the collision process have been introduced. As shown in Figure 3 and Figure 4, the energy lost by the wave-packet is commuted into kinetic (i.e. translational) and potential (i.e. collective vibrations) energies. Each of the two energy pools will be dissipated once the collision is completed, and a relaxation time can be defined for each process. The kinetic part of the energy of the particle, involving external DoF, will be dissipated against the liquid friction with a own decay time $\tau_{k}$, along the decay process

47. $\Delta E_{k}^{p}(t)=\Delta E_{k}^{p}(t=0) \cdot e^{-t / \tau_{k}}$.

The part responsible for the excitation of the internal collective vibratory (quantized) DoF will be relaxed along with the relaxation time(s) $\tau_{\Psi_{i}}$ pertaining to the several excited distinct DoF, and following the decay process [86]

48. $\quad \Delta \Psi_{i}^{p}(t)=\Delta \Psi_{i}^{p}(t=0) \cdot e^{-t / \tau_{\Psi_{i}}}$

As pointed out by many Authors [86-89], a distribution of relaxation times has been found in complex systems, as liquids, rather than a single relaxation time. However, being this specific topic outside the scope of the present paper, we still consider a single relaxation time $\left\langle\tau_{R}\right\rangle$ working for our system. We are indeed here only concerned with a comparison of the several time intervals introduced in our model with the wave-packet lifetime. Infact, depending on the length of the relaxation time $\left\langle\tau_{R}\right\rangle$, defined in Eqs.(7), (47) and (48), with respect to the wave-packet lifetime $\left\langle\tau_{w p}\right\rangle$, defined in Eq.(16), the dynamic iceberg may interact with another phonon before or after $\left\langle\tau_{R}\right\rangle$ has elapsed [this depends also on the wave-packets density in liquids, see Eq. (40)].

Experimental investigations performed in several liquids have shown that the scattering spectra are characterized by two distinct zones: the so-called viscous, or hydrodynamic-like regime, and the elastic, or solid-like regime [8-24], as foreseen also by numerical simulations [69]. Depending on the liquid nature, these branches may be explored in the sub-THz or THz frequency range. In all cases the spectra (INS or IXS) show a Positive Sound Dispersion, or simply PSD, i.e. an increasing value of the sound speed from low to high frequencies, or alternatively, from low to high wave- 
vectors; said in other words, PSD consists in a gradual increase of sound velocity upon increasing of the exchanged momentum $\Delta p$ or of the exchanged energy $\Delta \varepsilon$. This is shown in the two plots reported in Figure 5, where data extracted from [23] for water and from [10] for glycerol are reported. What immediately jumps to the eye is that the speed of sound spans from the hydrodynamic value, at low momenta, to the solid-like value, at high momenta. Incidentally, for water the solid-like value is that found for the first time by Ruocco et al in 1996 [15]. The propagation of acoustic, or elastic, waves is associated with the compression and rarefaction of the medium, or density fluctuations. At low frequencies, or low propagation speeds, the viscous regime is characterized by relaxation times much shorter that the period of oscillation of the wave packets $\tau=\left(\tau_{p}+\tau_{R}\right)<<\tau_{w p}=1 / v^{w p}$, where $v^{w p}$ is the wave-packet frequency [10, 23, see also Eqs. (7) and (16)]. This is equivalent to affirm that the target sample relaxes quickly, and the acoustic perturbation propagates over successive equilibrium states. On the contrary, at high frequencies and high propagation speeds, the elastic regime is characterized by periods of oscillation of elastic perturbations much shorter than the relaxation times, $\tau_{w p}<<\tau$. In this case the acoustic oscillations are too much quick to allow the DoF to relax their energy before a second perturbation hit the liquid particle again. In this regime the perturbations propagate elastically, with very much reduced energy loss. The intermediate branch, i.e. that in between the viscous and the elastic regimes, is usually referred to as the visco-elastic regime; it corresponds to the energy, or momentum range in which the liquid undergoes the transition from viscous to elastic response. This specific range corresponds to the occurrence of $\tau / \tau_{w p} \cong 1$, from which one may deduce the value of the relaxation time, $\tau \cong 1 / v^{w p}$. The increase of momentum and energy exchanged between phonons and liquid particles in the viscous-to-elastic transition or, what is the same, the decrease of acoustic dissipation, brings us to conclude that at low frequencies, or low sound velocity, the mechanism at the base of the wave packets $\leftrightarrow$ liquid particles interactions is mainly collisional and involves long wavelengths; at high propagation speeds, and therefore at high frequencies, the transmission-propagation mechanism of elastic waves involves also structural modifications, activating internal relaxation phenomena; in this case the interactions are characterized by short wavelengths. One may readily conclude that the coupling of a sound wave with a specific degree of freedom involves not only a characteristic time-scale, through the relaxation time, but also a length-scale, through the exchanged momentum, $\left\langle\Lambda_{p}\right\rangle \approx \lambda=2 \pi / q$ (indeed, for a wave-packet $\leftrightarrow$ liquid-particle interaction to occur, the wave-packet characteristic wavelength, or its extension, should be large at more as the particle size, if not smaller). From Figure 5 we see that, when a relaxation time due to internal DoF is active, the sound velocity depends also on the exchanged momentum or, equivalently, on the probed distance, as pointed out in [10, 23].

If the wave-packet $\leftrightarrow$ liquid-particle interactions would be perfectly elastic, it should be possible to calculate, at least in a simple schematic case, the OoM of particle displacement and of the lifetime of liquid particles between two successive interactions. In this case, all the energy (and momentum) lost by the wave-packet would be acquired by the particle and converted into kinetic energy, only the translational DoF being involved in the scattering process. In the specific case dealt with here, being the interaction anharmonic, it is not instantaneous; the momentum exchanged between wave-packet and particle is proportional to the duration of the interaction, $\left\langle\tau_{p}\right\rangle$ (i.e. to the time-length of the wave-packet) while the energy lost by a wave-packet in an event of type a) of Figure 3 is only partially converted into kinetic energy of the particle. The remaining part is converted into internal molecular energy, exciting the corresponding DoF. This is an important point of the model, which deals with for the first time the exchange of energy with the internal vibratory DoF of the molecular cluster in a phononic model of liquid. It is possible to define an 
"effective heat capacity" $C_{\text {eff }}$ or the total heat absorbed by (or given to) a liquid particle, taking into account the two branches of DoF. $C_{e f f}$ results to be a complex quantity whose real part is the static part pertaining to external translational DoF, while the imaginary part depends on that pertaining to the internal vibratory (quantized) DoF, on the frequency and on the relaxation time [86]. The pool of energy stored into the cluster will be released after a time lag $\left\langle\tau_{R}\right\rangle$. Depending on the OoM of such time interval, the events of Figure 3 can be considered elastic, quasi-elastic or inelastic $[11,25,34,73]$. Results of Section 3.4, already discussed at the beginning of the present Section, are in line with such argument, with the experimental outcomes and with the forecasts of PLT.

Which is the dynamics we imagine in a liquid? With reference to Figure 1, a wave-packet travels at the speed $u^{w p}=\left\langle\Lambda_{w p}\right\rangle /\left\langle\tau_{w p}\right\rangle$ until the border of a lattice. At this point there is the interaction described in Figure 3 and by Eqs. (4) and (5), in which energy and momentum are lost by the wave-packet to be acquired by the new domain. Part of this energy is commuted into kinetic energy of the cluster, part excites internal quantized DoF (harmonic contribution). The propagation of the perturbation inside the solid-like dynamic structure happens by means of (quasi) harmonic waves, travelling at speed $u^{0}=\left\langle\Lambda^{0}\right\rangle /\left\langle\tau^{0}\right\rangle$. The internal DoF will take a finite time to de-excite (Figure 4). As much longer will be this time with respect to the average lifetime $\left\langle\tau_{0}\right\rangle$ of the elastic perturbation inside the local lattice, so long will be $\left\langle\Lambda_{R}\right\rangle$. The change of order due to the collision between the wave packet and the liquid particle contributes to the specific heat, the thermal conductivity and some others liquid parameters. Such change of order requires energy to be added to, or taken from, the substance because of the change in potential energy associated with the change of structure. The time taken to such a change to occur is the relaxation time, and if the external change is too rapid, $\langle\tau\rangle>>\left\langle\tau_{w p}\right\rangle$, the required structure change cannot follow it. Said in other words, the system takes too much time to lose memory of its past with respect to the time taken by another external stimulus to occur. The relaxation time increases rapidly with decreasing the temperature. At temperatures where the relaxation time amounts to about 30 minutes, the order cannot adjust itself to equilibrium during the usual time an experiment takes. The momentary state of order is "frozen in" and the material is in the "glassy" state, which exists below the glassy transition temperature. The glassy state parameters are more like those of a solid than those of a liquid (in the classical meaning). The glass has shear rigidity because the usual time of stress application is much shorter than the shear relaxation time. The experiments performed in the visco-elastic regime of liquids have shown that such behaviour persists in liquids for stresses of the duration of picoseconds. Therefore, in their behaviour in propagating ultrasonic waves, liquids may become "glassy" at much higher temperatures (shorter relaxation times) than for ordinary experiments. They then behave like solids on short length scales, giving little absorption and showing, for transversal waves, shear rigidity. Short relaxation times, $\langle\tau\rangle\left\langle\left\langle\left\langle\tau_{w p}\right\rangle\right.\right.$, indicate phenomena that relax before a liquid particle has undergone a second interaction. In this case the wave-packet will see in the second interaction a system that is modified (relaxed) with respect to the first collision. In the opposite case, $\langle\tau\rangle>>\left\langle\tau_{w p}\right\rangle$, the wave-packet shall find a system unmodified with respect to the first event, as in a rigid network [29,10].

A question that the reader has certainly asked himself at this point is whether it is possible to give an expression for the lattice potential that gives rise to the interaction term $f^{\text {th }}$. An even more fundamental question is whether 
phonon-particle collisions can be treated as a classical scattering or it is necessary to examine them from a quantum point of view to analyze the consequences of energy absorption and re-emission, in particular for the internal collective vibratory DoF. To answer this question, let us consider the motion of a free particle, for instance a single electron. Its motion is not quantized in the external DoF, but only in the spin. However, if this particle is immersed in an external force field, then its motion will be quantized too, as for example an electron in the Coulombian field generated by a positive charge, as in the hydrogen atom. It will not be able to travel any orbits, but only those which satisfy certain conditions dictated by the rules of quantum mechanics. These conditions also limit the translational DoF of the electron; in fact the energy, the total angular momentum (modulus) and the azimuthal component are quantized. Of course, this does not exclude that an isolated atom can rotate freely on itself. If, however, this atom is in turn immersed in a force field, for example because it is linked to other atoms of a molecule or of a larger lattice, then its global motion will also be quantized compared to the other atoms of the same system. His internal rotational and vibrational DoF will then also be quantized. This reasoning, as is known, can be extended to even larger systems, at least within the limits imposed by the Heisenberg principle, which delimits the transition from the quantized world to the classical one. The statistical nature of the wave packet -liquid particle interactions does not allow in its current formulation a hypothesis about the lattice potential of a liquid, so that the two populations, phonons and liquid particles, which make up the liquid, are in fact supposed not interacting at a distance, but only when there is an overlap of the respective wave packets, from which the origin of the finite interaction time $\left\langle\tau_{p}\right\rangle$. The dynamic effect is generated only during the interaction itself, although the two subsystems are not a-priori brought to interact, like two particles driven by a potential. One may wonder if this fact could be an indication of the short-range character of the lattice potential. In the DML the "contact" character of the interaction leads to suppose the liquid particles as "free" before and after the interaction. Based on this hypotheses, it is plausible to assume the translational and rotational DoF of the entire particle as non-quantized (if the links with the other liquid particles are neglected). Therefore, only the internal vibratory DoF would remain quantized.

Some considerations are in order about the similitude between the DML and the PLT models. The PLT describes the liquid from a statistical-thermodynamic point of view, therefore it cannot provide a direct answer on "why" the liquid particles oscillate. DML instead faces the problem from the opposite, mesoscopic point of view, starting just from the elementary wave packet $\leftrightarrow$ liquid particle collision that gives rise to the harmonic and anharmonic contributions even present in the PLT. DML provides and analyzes the microscopic elementary mechanism by which elastic and thermal energy, as well as momentum, are exchanged between liquid particles (icebergs) and lattice particles (phonons) and show that this scenario is in agreement with that of the PLT. One of the junction points between the two approaches, PLT and DML, is in fact just the relaxation time. Because of the thermodynamicstatistical nature of the theory, PLT from its side is only able to provide an indication of the variation range of the relaxation time through the value of the product $v \tau$, as discussed above. On the other side, DML is a mesoscopic model and analyzes the intimate mechanism of interaction of liquid particles with lattice modes; this provides both the answer to the question of "why" liquid particles oscillate, and a way for calculating the order of magnitude of $\tau$ in ordinary liquids. We have shown above that the attained value of $\tau$ and of the product $v \tau$ are in the range foreseen on thermodynamic statistical bases from PLT. The relaxation time is one of the key-points of both models, and because the only common argument of PLT and DML is the assumption that thermal energy in liquids is transported by means of collective lattice excitations, both harmonic and anharmonic, the mutual agreement shows that both of them look at the model of liquid state in the same way, although from different observation points. 
Another similitude of PLT and DML is the presence of harmonic and anharmonic contributions to the pool of energy. From the statistical-thermodynamic point of view, this fact is accounted for in the PLT by considering both contributions into the Hamiltonian of a liquid, giving rise in turn to the expression for the specific heat (the Hamiltonian adopted in the PLT has however many other interesting consequences, which anyway are not discussed here being out of the specific topic of the present paper). On the other side, harmonic and anharmonic oscillations are believed in the DML responsible for the propagation of energy and momentum within a liquid. In fact, energy is supposed to propagate inside the solid-like aggregates by means of harmonic oscillations. Liquid particles communicate among them and with the disordered liquid by means of anharmonic wave-packets, capable as such to exchange not only energy but also momentum. In such a way the macroscopic phenomena of diffusion (and thermal diffusion) may be easily interpreted in the DML.

The duality of liquids, supposed by both DML and PLT, however, does not exclude of course classical mechanisms of intermolecular interactions. The propagation of energy in a condensed medium through inter-molecular interactions on one side, or interactions between wave packets and pseudo-crystalline structures on the other, may be supposed working simultaneously and uniformly at the mesoscopic level. Which of the two prevails over the other depends on the thermodynamic conditions of the system. In a solid only the phononic part will be present. When the solid melts, the molecules begin to arrange in local solid-like lattices, phononic propagation gradually leaves way for propagation through molecular interactions, until it completely disappears when the gaseous state is reached, only the molecular one remaining. While in solids there are always three modes of vibration, two transversal and one longitudinal, in liquids the transverse modes will be active only for frequencies higher than $\omega_{F}, \omega>\omega_{F}=\frac{2 \pi}{\tau_{F}}$, where $\tau_{F}$ is the relaxation time. In other words, on interaction times lower than the relaxation time, liquids behave as solids, while for times longer than $\tau_{F}$, liquids lose the ability to support transversal ways, as in the classical approach. This view in the DML agrees with the interpretation underlying the Positive Sound Dispersion (PSD) (see Figure 5). The propagation speed of elastic and thermal waves in liquids reaches values close to those in the corresponding solids as the frequency increases. On a macroscopic scale, at low frequencies, and therefore large wavelengths, it is not possible to reveal the presence of the local lattices, while they show their presence when the wavelengths become comparable to, or smaller than, the size of such clusters. As consequence of the above, the number $m$ of lattice collective DoF available in a liquid, introduced in Eq.(15), decreases as the temperature increases, $d m / d T<0$. At low temperature, when the two transversal modes and the longitudinal one are excited, $m$ approaches unity, while it approaches to zero at high temperatures because the number of lattice collective DoF decreases with increasing $\mathrm{T}$, $0 \leq m \leq 1$, until they completely disappear (at the Frenkel line). This is a fundamental point of the DML. In a separate paper [83], this argument is deeply discussed and limits for $d m / d T$ and $m$ are deduced and analysed.

The evaluation of the crucial parameters rests then on that of $\left\langle\tau_{0}\right\rangle,\left\langle\tau_{p}\right\rangle$ and $\left\langle\tau_{R}\right\rangle$, and hence on the physical modelling of the relaxation processes involving internal DoF $[11,34,73]$. An interesting theory has been proposed by Nettleton [87-89] that introduced a set of phenomenological equations, valid at all frequencies, for the rates of change of internal relaxation parameters describing molecular excitations and structural changes in a liquid. He found an Onsager coupling between the rate equations and the pressure tensor. 
The values of phonon mean-free-path $\left\langle\Lambda_{0}\right\rangle$ and life-time $\left\langle\tau_{0}\right\rangle$ inside a liquid particle can be deduced from the experimental values obtained in light scattering experiments. Indeed $\left\langle\Lambda_{0}\right\rangle$ will be a multiple of the phonon wavelength $\lambda^{0},\left\langle\Lambda_{0}\right\rangle=n \lambda^{0}$, and $\left\langle\tau_{0}\right\rangle$ of $\tau=1 / v^{0},\left\langle\tau_{0}\right\rangle=n / v^{0}$, with $n>1$. Using for instance the data for water of $[10,23]$ (the amount of data available in literature is very extensive, especially for water), typical values for the parameters characterizing a phonon (variation range is function of temperature, pressure and $q$ orientation) are: (central) frequency $\frac{n}{\left\langle\tau_{0}\right\rangle}=\left\langle v^{0}\right\rangle \approx 0,95 \div 2,5 \mathrm{THz}$, wave-length $\frac{\left\langle\Lambda_{0}\right\rangle}{n}=\left\langle\lambda^{0}\right\rangle \approx 1 \div 3 \mathrm{~nm}$ and velocity $\frac{\left\langle\Lambda_{0}\right\rangle}{\left\langle\tau_{0}\right\rangle}=\left\langle\lambda^{0}\right\rangle \cdot\left\langle v^{0}\right\rangle=\left\langle u^{0}\right\rangle \approx 3.1 \div 3.4 \cdot 10^{3} \mathrm{~m} / \mathrm{s}$. Interestingly, this value fits very well the experimental data obtained for the propagation velocity of thermal waves in water [15].

The deduced value for $\left\langle\Lambda_{0}\right\rangle$ gives us also the possibility to evaluate the size of a "liquid particle". Consequently, we may argue that its typical size is of the same OoM as $\left\langle\Lambda_{0}\right\rangle$ at the exchanged momentum $q$. We may then confidentially assume a value of few units for the parameter $n$.

Regarding the possibility of thinking to the external temperature gradient as the generalized force, the energy flow generated by it is modelled as a variation of the wave-packet energy, having neglected so far any variation in their density (although in principle also a density variation could be taken in consideration). The effect due to the local, or virtual, temperature gradient is different, it could be responsible for a local curvature of the lattice, which in turn is responsible for the phonon-liquid particle interaction. This is an interesting topic that will be further dealt with in a future work.

\section{Conclusions.}

In the Dual Model of Liquids the inertial term $f^{\text {th }}$ provides a physical mechanism by which a cluster of molecules and a liquid lattice exchange energy and momentum. The elementary interaction shown in Figure 3, besides being anharmonic and allowing momentum exchange, has the important property of being temporally reversible, as the non-equilibrium thermodynamics requires. In this frame, the experimental evidences obtained in the study of collective dynamics of liquids by means of INS and IXS techniques find a physical interpretation. Besides, the model is also able to postdict the physical interpretation of some parameters typical of liquids and of other well known phenomena. DML provides also a comparison with some results obtained by independent research groups on the dynamics and thermodynamics of liquid media (liquid specific heat and thermal conductivity, sound velocity, capability of transporting momentum, etc.).

The huge amount of experiments performed in controlled isothermal conditions [8-11, 13-37, 36, 55-56] allowed the discovery of the collective dynamics of liquids and the evaluation of sound velocity at high frequencies, that in water is close to that of ice. This aspect is interpreted within the DML with the presence in liquids of solid-like dynamic clusters of molecules in which elastic wave packets propagate as in the corresponding solid phase. The size of these cluster is the wave-packets mean free path, and they result to be made of 10 to 20 liquid molecules. The 
comparison of the relaxation time(s) and of the average lifetime of the wave-packets concurs towards the same vision. The number density of phonons, of the same OoM as the molecular density, also concurs with the hypothesis of single phonon-liquid particle interaction.

One of the peculiarities of the interactions between wave packets and liquid particles is the ability to move quantities of energy from one place to another in a well-defined time interval, the relaxation time, during which it is kept out of the heat current. The role of relaxation times is important in all the situations in which transport phenomena are influenced by a delay occurring in the microscopic transport processes. One of these cases is that of the heat transport in non stationary conditions, where such delays have a relevant role in the correct identification of the physical process and of the related mathematical equations describing the heat propagation in condensed media. It is well known indeed that in non stationary conditions a Cattaneo-like hyperbolic equation should be used instead of a Fourier-like parabolic one to describe the heat propagation. In a separate paper [72] it is shown how the DML allows to justify from a physical point of view the delay time appearing in the Cattaneo equation, proposed to replace the well-known Fourier equation.

Performing experiments in non-stationary temperature gradients may allow to investigate how liquid parameters evolve from equilibrium to non equilibrium conditions. It could be possible for instance to investigate the evolution of correlations lengths, sound velocity, thermal conductivity, etc.. Also very interesting could be the investigation of whether and how a temperature gradient affects the viscous coupling between two liquids. Such experiments could be performed, for instance, by applying a temperature gradient to a stabilised isothermal liquid, provided that the average temperature of the system remains unchanged and preventing the convection instability, and performing light scattering experiments until a stationary temperature gradient is reached, i.e. during the transient, exactly the situation that is normally avoided in all the experiments. An alternative way to heat a small volume of liquid could be that on hitting the liquid with a focalised high power laser beam.

A second type of experiments could be aimed at investigating the glassy and liquid-to-solid transitions. Starting from a stationary temperature gradient, both temperatures could be lowered, thus lowering also the average temperature until the liquid solidifies. Light scattering experiments performed during the non stationary phase should allow to investigate the dynamics of the system when the glassy and liquid-to-solid transitions are crossed. In particular, the local domains should be oriented following the external temperature gradient, thus allowing the increase of the correlation lengths, of sound velocity, of thermal conductivity, etc., along the preferential direction of the external temperature gradient. A difference between the same parameters when measured along the direction of the temperature gradient with respect to those measured along a different direction should be evident from the experimental data collected. This technique could in some way introduce a sort of anisotropy into the liquid matrix, becoming the solid phase anisotropic.

The results obtained by Esposito [84] and Nicolis [90] about the capability of phonons to carry negative mass could be experimentally confirmed by performing two types of experiments; one on ground in a system with an upward temperature gradient to avoid convection; the second in weightlessness, as for instance on orbiting platforms or, even better, in satellites orbiting in the Lagrange point. The latter would allow to measure the net effect of mass carried by phonons without the influence of gravity (in such a system the Clausius Postulate is valid for sure). Having a more clear vision from experiments of how mass and momentum are carried by phonons, may indeed help to understand how to manage their contribution in theoretical studies, and probably answer some of the questions raised in the previous section. 
The importance of thermal fluctuations has been recognized since a long time in the dynamics of proteins [see for instance 91-93] and the correlation between the high-frequency dynamics of liquid water and that of protein dynamics should be further investigated. It is also reasonable to expect that gradients of concentrations may give similar results as those due to temperature gradients. Performing such experiments in the study of protein crystal growth might allow to have an idea of the lengths on which aggregation starts and, probably, discover that temperature gradients may help in controlling and addressing correctly the crystal formation.

What we may finally deduce from above is that theory and experiments on the dynamics of liquid state give now a clear answer on the historical question on whether liquids have to be considered as condensed gases or molten solids, answer already anticipated by Brillouin in 1936 [4]: “On est donc contraint d'admettre un point de vue intermédiaire: un liquide, à basse température, serait un réseau cristallin défini, mais présentant une ou plusieurs directions de plans de clivage infinement facile. La distinction, par rapport au liquid idéal, est essentielle: nous aurons, dans de trés petits domaines, des structures cristallines; ces cristaux résistant à un effort tangentiel, si celui-ci s'exerce dans une direction autre que cell de clivage infinement facile." ... "Microscopiquement, (les liquides) sont des solides; macroscopiquement ils apparaitront liquides."

\section{Acknowledgments.}

The author wants to dedicate this paper in memoriam of prof. F.S. Gaeta, who recently passed away. The author wants even to express his gratitude to prof. F.S. Gaeta for his enlightening mentoring. At the same time, the author is grateful to prof. A. Romano for having read the manuscript and for the helpful discussions. 


\section{Figures}

Figure 1

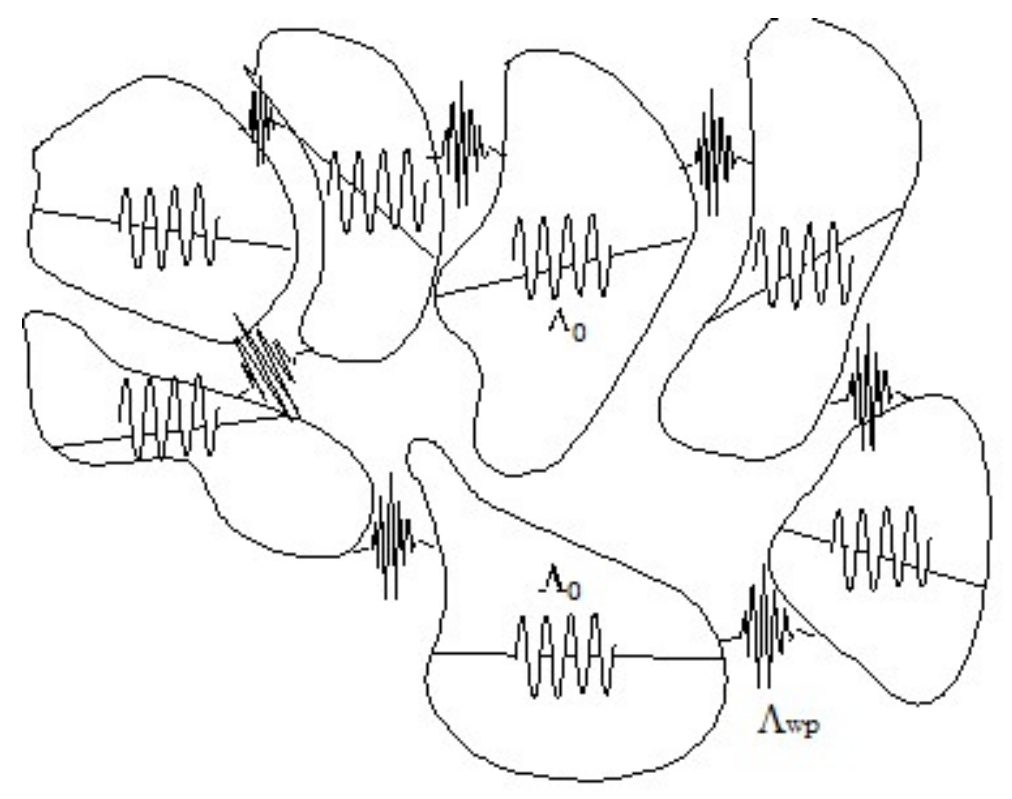

Figure 1. Dynamic solid-like structures fluctuating and interacting within the liquid global system at equilibrium. As far as thermal (elastic) perturbations propagate within such structures, they behave as in solids. Propagation velocity has then the values typical as those of the solid lattice, as found by Ruocco et al. [15] of about $3200 \mathrm{~m} / \mathrm{s}$ for the case of water. Average sizes of icebergs $\left\langle\Lambda_{0}\right\rangle$ have been found of some nanometers. When perturbations cross the boundary between two solid-like aggregates, $f^{\text {th }}$ develops and energy and momentum are transmitted from one to the nearestneighbour aggregate. This pictorial model of liquids at mesoscopic scale, on which the DML is based, reflects also what may be deduced from experiments performed with IXS techniques, able to observe liquids at such scale-lengths.

In a solution solute particles may be considered as solid-like aggregates having elastic impedance different from that of the solvent. Energy and momentum exchanged between the two types of these dynamic icebergs produce a net effect resulting in the diffusion of the solute along the concentration gradient. If a temperature gradient is imposed externally, the net effect will depend on the prevailing flux of wave packets, which will give rise to thermal diffusion of one species with respect to the other. 
Figure 2

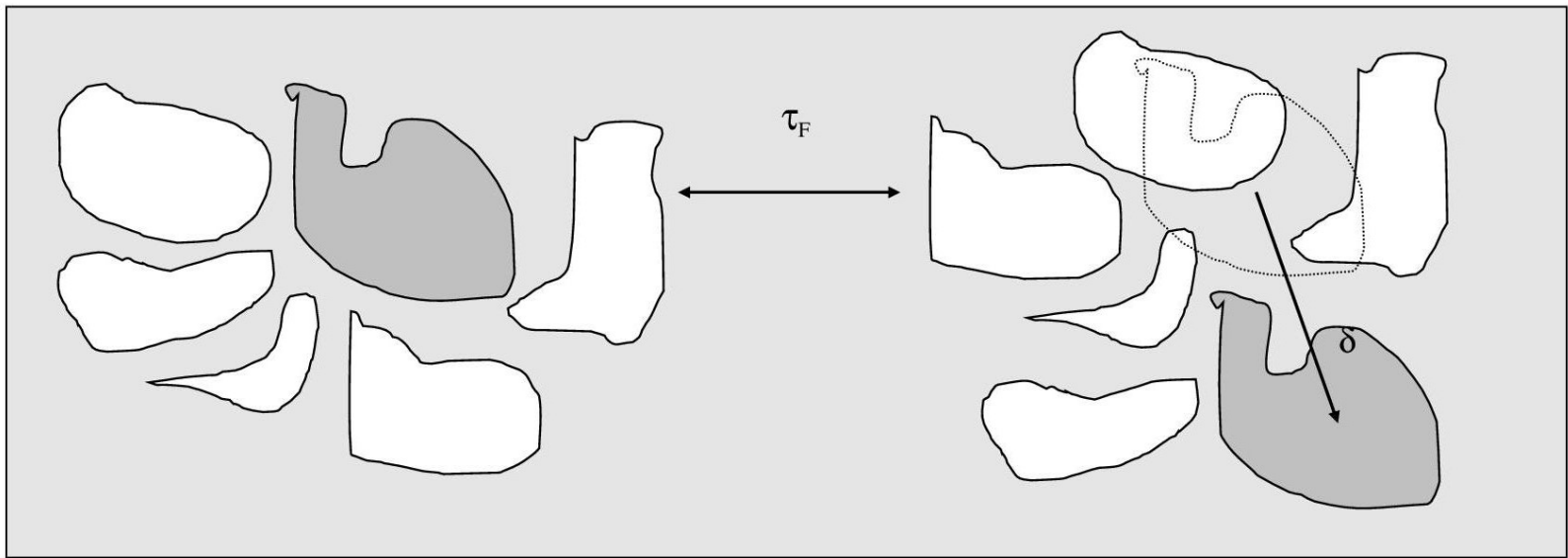

Figure 2. Illustration of a "wandering iceberg" jumping between two equilibrium positions in a liquid. The jump takes place after an average relaxation time lasting $\tau_{F}$, during which the liquid particle moves by a distance $\delta$. 
Figure 3
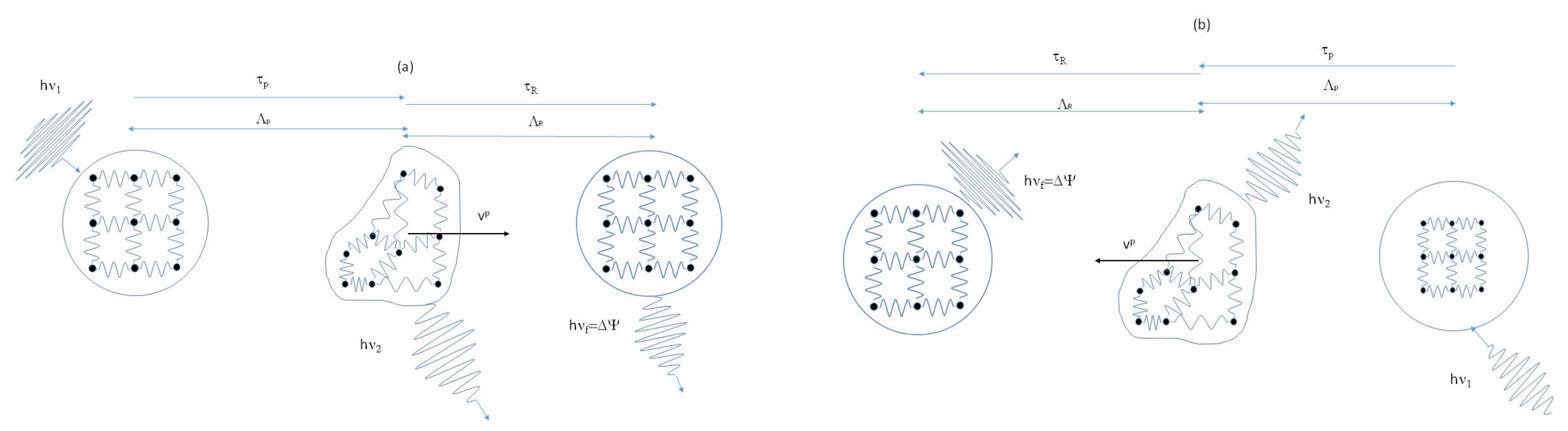

$$
\Delta \varepsilon^{w p}=h\left\langle v_{1}\right\rangle-h\left\langle v_{2}\right\rangle=\Delta E_{k}^{p}+\Delta \Psi^{p}=f^{t h} \cdot\left\langle\Lambda_{p}\right\rangle \quad \Delta p^{w p}=f^{t h} \cdot\left\langle\Lambda_{p}\right\rangle
$$

Figure 3. Schematic representation of wave-packets and liquid particles inelastic collisions. The event represented in (a), in which an energetic wave-packet transfers energy and momentum to a liquid particle, is commuted upon time reversal into the one represented in (b), where a liquid particle transfers energy and momentum to a wave-packet. The particle changes velocity and the frequency of wave-packet is shifted by the amount $\left(v_{1}-v_{2}\right)$. The interaction lasts $\left\langle\tau_{p}\right\rangle$ during which the particle moves by $\left\langle\Lambda_{p}\right\rangle$; the relaxation lasts $\left\langle\tau_{R}\right\rangle$ during which the particle moves by $\left\langle\Lambda_{R}\right\rangle$. Due to its time symmetry, this mechanism is assumed the equivalent of Onsager' reciprocity law at mesoscopic level. In a pure isothermal liquid energy and momentum exchanged among the icebergs are statistically equivalent, and no net effects are produced. Events of type a) will alternate with events of type b), to maintain the balance of the two energy pools unaltered. Besides, the macroscopic equilibrium will ensure also the mesoscopic equilibrium; events (a) and (b) will be equally probable along any direction, to have a zero average over time and space. On the contrary, if a symmetry breaking is introduced, as for instance a temperature or a concentration gradient, one type of event will prevail over the other along a preferential direction. 


\section{Figure 4}

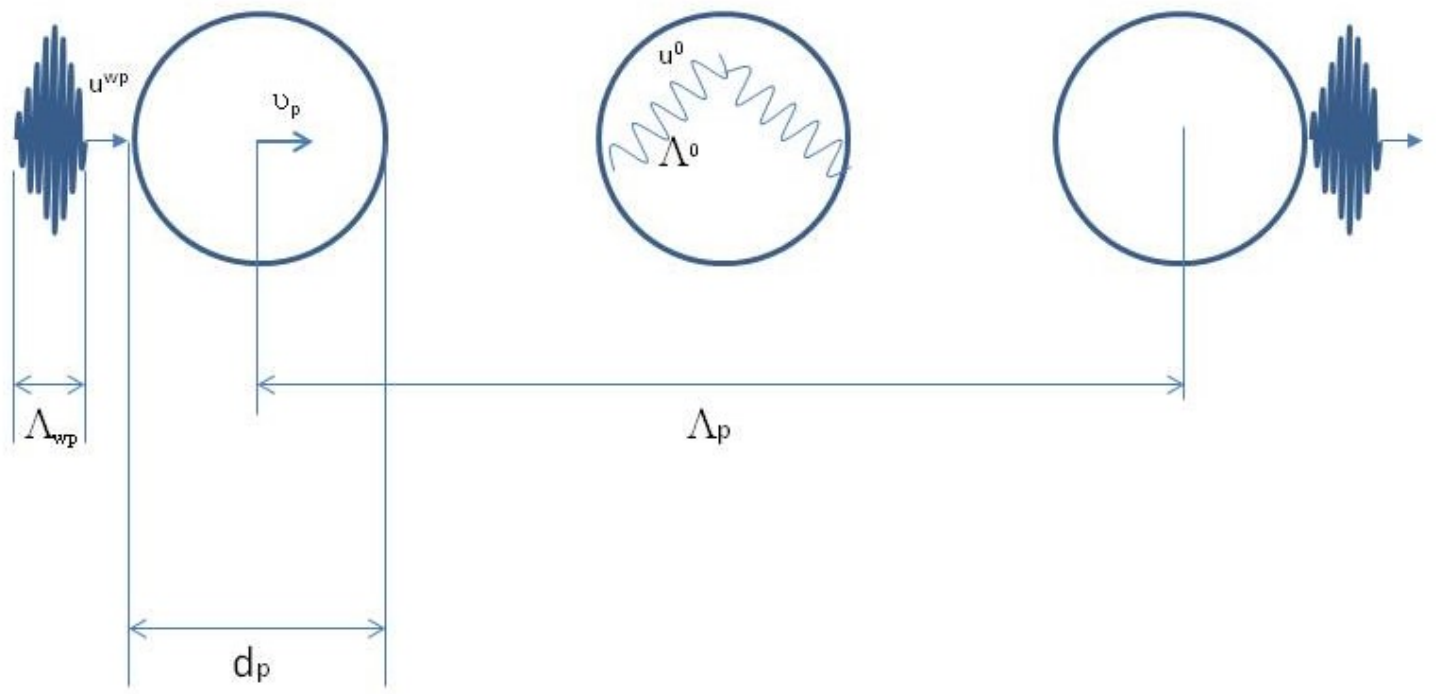

Figure 4. Close-up of the first part of the wave-packet - particle interaction shown in Figure 3a, during which the wave packet transfers energy and momentum to the liquid particle. $\Lambda_{w p}$ is the extension of the wave-packet, and $d_{p}$ that of the liquid particle. Once $\tau_{p}$ has elapsed and the liquid particle has travelled by $\Lambda_{p}$, the particle relaxes the energy stored into internal DoF; then it travels by $\Lambda_{R}$ during $\tau_{R}$ (not shown in the figure above). 
Figure 5
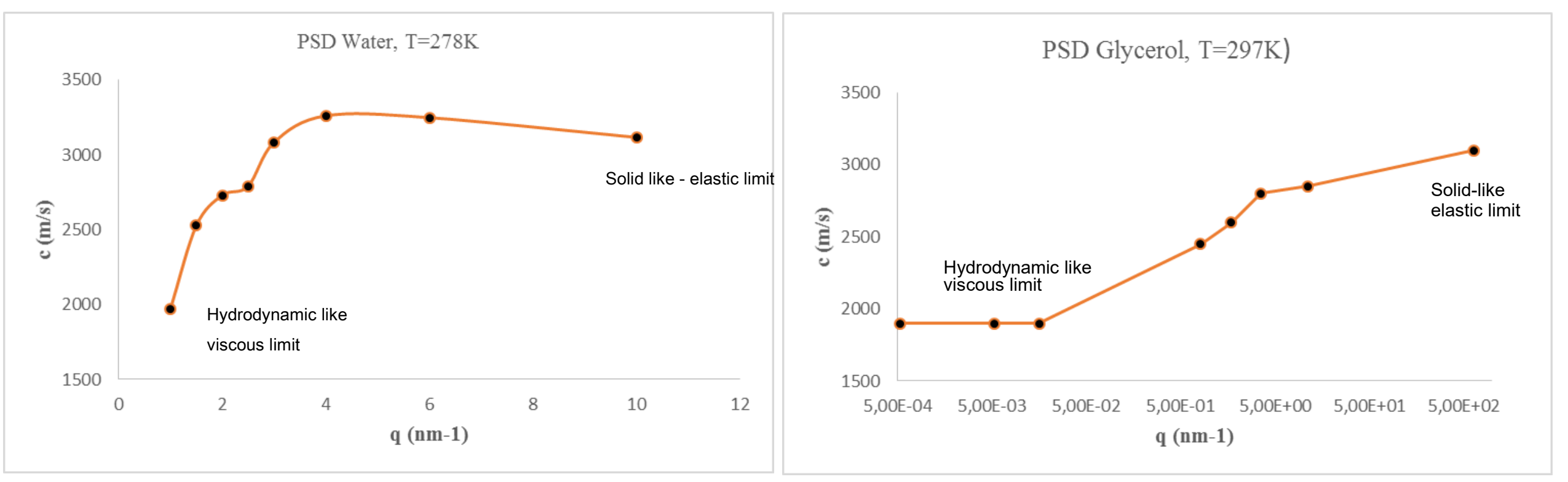

Figure 5. Positive Sound Dispersion - PSD - for Water and Glycerol at the indicated temperature and momentum values. Data are from [23] for Water and from [10] for Glycerol. 


\section{Tables}

Table 1

\begin{tabular}{|c|c|c|}
\hline Parameters & Variables & Eqs.(\#) \\
\hline \hline$\eta_{l}=9 \cdot 10^{-4} \mathrm{~Pa} \cdot s$ & $35 \leq v_{p} \leq 176 \mathrm{~m} / \mathrm{s}$ & 31 \\
\hline$v^{w p}=0.9 ; 1.0 ; 1.5 ; 2.0 ; 3.0 \cdot 10^{12} \mathrm{~Hz}$ & $0.29 \leq \Lambda_{p} \leq 1.20 \cdot 10^{-9} \mathrm{~m}$ & 39 \\
\hline$\alpha=0.3 ; 0.5 ; 0.7$ & $4.0 \leq \tau_{p} \leq 13.0 \cdot 10^{-12} \mathrm{~s}$ & 35 \\
\hline$n=5$ & $8.9 \leq \Delta^{p h} \leq 22.9{ }_{6} \cdot 10^{-9} \mathrm{~m}$ & 32 \\
\hline$m_{p}=3 ; 5 ; 10 m_{\mathrm{H}_{2} \mathrm{O}}=9 ; 15 ; 30 \cdot 10^{-26} \mathrm{~kg}$ & $1.3 \leq \tau_{\eta} \leq 8.8 \cdot 10^{-15} \mathrm{~s}$ & 42 \\
\hline$r_{p}=1.0,1.5,2.0 \cdot 10^{-9} \mathrm{~m}$ & $0.045 \leq \Lambda_{\eta} \leq 1.5 \cdot 10^{-12} \mathrm{~m}$ & 43 \\
\hline
\end{tabular}

Table 1. List of parameters $\left(1^{\text {st }}\right.$ column $)$ introduced to evaluate the several variables $\left(2^{\text {nd }}\right.$ column $)$ characterizing the mesoscopic dynamics of liquids, and of the corresponding values for the named parameters. In the $3^{\text {rd }}$ column the number of equation is listed that has been used to calculate the variables listed in the $2^{\text {nd }}$ column. 


\section{References}

1. Debye, P., 1912, Zur Theorie des specifische Wärmer, Ann. der Physik, 344 Nr.14, 798-839.

2. Debye, P., 1914, Vorträge über die Kinetische Gastheorie, pp 46-60, Leipzig, B.G. Teubner.

3. Brillouin, L., 1922, Ann. de Phys., 17, 88.

4. Brillouin, L., 1936, La chaleur spécifique des liquides et leur constitution, J. Phys. Rad, Serie VII, Tome VII, N. 4, 153-157

5. Frenkel, J., 1946, Kinetic theory of liquids, Oxford, Oxford University Press.

6. Croccolo, F., Ortiz de Zarate, J.M., Sengers, J.V., 2016, Non local fluctuations phenomena in liquids, Eur. Phys. J. E, 39, 125-136.

7. Bolmatov, D., Trachenko, K., 2011, Liquid heat capacity in the approach from the solid state: Anharmonic theory, Physical Review B, 84, 054106

8. Ruocco, G., Sette, F., 2008, The history of fast sound in liquid water, Cond. Matt. Phys., 11, No 1(53), 29-46

9. Cunsolo, A., 2012, Onset of a transverse dynamics in liquid water, Molecular Physics, 111 Nr. 3, 455-463.

10. Cunsolo, A., 2015, The terahertz spectrum of density fluctuations of water: the viscoelastic regime, Adv Cond. Matt. Phys., 2015, 137435-137459.

11. Cunsolo, A., 2017, The terahertz dynamics of simplest fluids probed by X-ray scattering, International Review in Physical Chemistry, 36-3, 433-539.

12. Cunsolo, A., 2017, Inelastic X-ray scattering as a probe of the transition between the hydrodynamic and the single particle regimes in simple fluids., http://dx.doi.org/10.5772/66126.

13. Sette, F., Ruocco, G., Krisch, M., Bergmann, U., Masciovecchio, C., Mazzacurati, V., Signorelli, G., Verbeni, R., 1995, Collective dynamics in water by high-energy resolution inelastic X-ray scattering, Phys. Rev. Lett., 75, $850-854$.

14. Sette, F., Ruocco, G., Krisch, M., Masciovecchio, C., Verbeni, R., 1996, Collective dynamics in water by inelastic X-ray scattering, Physica Scripta, T66, 48-56.

15. Ruocco, G., Sette, F., Bergmann, U., Krisch, M., Masciovecchio, C., Mazzacurati, V., Signorelli, G., Verbeni, R., 1996, Equivalence of the sound velocity in water and ice at mesoscopic lengths, Nature, 379, 521-523.

16. Sette, F., Ruocco, G., Krisch, M., Masciovecchio, C., Verbeni, R., Bergmann, U., 1996, Transition from normal to fast sound in liquid water, Phys. Rev. Lett., 77, 83-86

17. Ruocco, G., Sette, F., Krisch, M., Bergmann, U., Masciovecchio, C., Verbeni, R., 1996, Line broadening in the collective dynamics of liquid and solid water, Phys. Rev. B, 54, 14892-14895.

18. Sampoli, M., Ruocco, G., Sette F., 1997, Mixing of longitudinal and transverse dynamics in liquid water, Phys. Rev. Lett., 79, 1678.

19. Sette, F., Krisch, M., Masciovecchio, C., Ruocco, G., Monaco, G., 1998, Dynamics of glasses and glass-forming liquids studied by inelastic X-ray scattering, Science, 280, 1550-1555.

20. Ruocco G., Sette, F., The high-frequency dynamics of liquid water, 1999, J. Phys. Cond. Matt, 11, R259-R293.

21. Monaco G., Cunsolo A., Ruocco G., Sette F., 1999, Viscoelastioc behaviour of water in the THz frequency range: an inelastic X-ray study., Phys. Rev.E, 60-5, 5505-5521.

22. Scopigno, T., Balucani, U., Rocco, G., Sette, F., 2002, Inelastic X-ray scattering and the high-frequency dynamics of disordered systems, Physica B, 318, 341-349 
23. Cunsolo A., Ruocco G., Sette F., Masciovecchio, C., Mermet, A., Monaco, G., Sampoli, M., Verbeni, R., 1999, Experimental determination of the structural relaxation in liquid water, Phys. Rev. Lett., 82-4, 775-778.

24. Cunsolo, A., Inelastic X-Ray scattering as a probe of the transition between the hydrodynamic and the singleparticle regimes in simple fluids, http://dx.doi.org//10.5772/66126

25. D. Bolmatov, D., Brazhkin, V.V., Trachenko, K., 2012, The phonon theory of liquid thermodynamics, Scientific Reports, 2, 431-427 (DOI:10.1038/srep00421.)

26. Brazhkin, V.V., Trachenko, K., 2012, What separates a liquid from a gas?, Phys. Today, 65(11), 68-69; doi:10.1063/PT.3.1796.

27. Bolmatov, D., Brazhkin, V. V., Fomin, Yu. D., Ryzhov, V. N., Trachenko, K., 2013, Evidence for structural crossover in the supercritical state, Journal of Chemical Physics, 139, 234501.

28. Trachenko, K., Brazhkin, V.V., 2013, Duality of liquids, Scientific Reports, 3, $2188-2193$ (DOI: 10.1038/srep02188).

29. Bolmatov, D., Musaev, E. T., Trachenko, K., 2013, Symmetry breaking gives rise to energy spectra of three states of matter, Scientific Reports, 3, 2794.

30. Bolmatov, D., Zhernenkov, M., Zav’yalov, D., Tkachev, S. N., Cunsolo, A., Cai, Y. Q., 2015, The Frenkel Line: a direct experimental evidence for the new thermodynamic boundary, Scientific Reports, 5, 15850.

31. Bolmatov, D., Zhernenkov, M., Zav'yalov, D., Stoupin, S., Cunsolo, A., Cai, Y. Q., 2016, Thermally triggered phononic gaps in liquids at THz scale, Scientific Reports, 6, 19469.

32. Bolmatov, D., Zav'yalov, D., Zhernenkov, M., Musaev, E.T., Cai, Y.Q., 2015, Unified phonon-based approach to the thermodynamics of solid, liquid and gas states, Ann. Phys., 363, 221-242.

33. Bolmatov, D., Zhernenkov, M., Zav'yalov, D., Stoupin, S., Cai, Y.Q., Cunsolo, A., 2015, Revealing the mechanism of the viscous-to-elastic crossover in liquids, J. Phys. Chem. Lett., 6, 3048-3053 (doi: 10.1021/acs.jpclett.5b01338).

34. Trachenko, K., Brazhkin, V.V., 2016, Collective modes and thermodynamics of the liquid state, Rep. Prog. Phys. 79, 016502-016538.

35. Balucani, U., Brodholt, J.P., Vallauri, R., 1996, Dynamical properties of liquid water, J. Phys. Cond. Matt., (8)47, 9269-9274.

36. Balucani, U., Ruocco, G., Torcini, A., Vallauri, R., 1993, Fast sound in liquid water, Phys. Rev. E, 47-3, 16771684.

37. Teixeira J., Bellisent-Funel, M.C., Chen, S.H., 1985, Observation of new short-wavelength excitations in heavy water by coherent inelastic neutron scattering, Phys. Rev. Lett., 54, 2681-2683.

38. Sköld, K., 1967, Small energy transfer scattering of cold neutrons from liquid argon, Phys. Rev. Lett., 19, 10231025 DOI: http://dx.doi.org/10.1103/PhysRevLett.19.1023.

39. Fiks, V.B., 1961, On the thermodiffusion mechanism in fluids, Soviet Physics-Solid State, 3, 724-726.

40. Fiks, V.B., 1964, Electron drag and thermal diffusion in metals, Soviet Physics-Solid State, 5, $2549-2552$.

41. Andreev, A.F., 1971, Two-Liquid effects in a normal liquid, JEPT, 32-5, 987-990

42. Gaeta, F.S., 1969, Radiation pressure theory of thermal diffusion in liquids, Phys. Rev. A, 182, 289-296.

43. Gaeta, F.S., Peluso, F., Mita, D.G., Albanese, C., Castagnolo, D., 1993, Phonon-particle interactions and transport processes in liquids, Phys. Rev. E, 47, 1066-1077.

44. Gaeta, F.S., Albanese, C., Mita, D.G., Peluso, F., 1994, Phonons in liquids, Onsager's reciprocal relations and the heats of transport, Phys. Rev. E, 49, 433-444. 
45. Gaeta, F.S., Mita, D.G., 1978, Nonisothermal mass transport in porous media, J. Membrane Sci., 3191.

46. Gaeta, F.S., Mita, D.G., 1979, Thermal diffusion across porous partitions: the process of thermodialysis, J. Phys. Chem., 832276.

47. Zwanzig, R., 1961, in Lectures in theoretical physics, edited by W. Brittin (Wiley-Interscience, New York).

48. Mori, H., 1965, A continued-fraction representation of the time-correlation functions, Prog. Theor. Phys., 34, 399-416

49. Lucas, M.R., 1938, Sur l'agitation thermique des liquides, leur nouvelles propriétés thermomécahnique et leur conducibilité calorifique, J. Phys. 10, 410-428

50. Landau, L., Placzek, G., 1934, Z. Phys. Sowjetunion, 5, 172; Landau, L., 1986, Elettrodinamica dei mezzi continui, (Moscow, MIR), § 120.

51. Fabelinskii, I., 1968, Molecular scattering of light, (New Yor, Plenum Press), Chap. 2, § 5

52. Berne, B., Pecora, R., 1976, Dynamic Light Scattering, New York, John Wiley \& Sons.

53. Egelstaff, P.A., 1967, An Introduction to the Liquid State, chap. 10, London \& New York, Academic Press.

54. Hansen, J.-P., McDonalds, I.R., 2013, Theory of Simple Liquids, IV Ed., Amsterdam, Elsevier.

55. Meyer, E.H.L., Ramm, W., 1932, Die Strukture der Rayleigh Strahlung Physik Zeitschr., 33, 270.

56. Raghavendra-Rao, B.V., 1934, Examination of molecular scattered light with a Fabry-Perot etalon, Proc. Ind. Acad. Sci., 1, 261

57. see for instance Grimwall, G., 1975, Physica Scripta 11, 381.

58. Kirkpatrick, T., Cohen, E.G.D., Dorfman, J.R., 1979, Kinetic theory of light scattering from a fluid not in equilibrium, Phys. Rev. Lett., 42862.

59. Kirkpatrick, T., Cohen, E.G.D., 1980, Light scattering in a fluid far form equilibrium, Phys. Lett., 78A, 350.

60. Kirkpatrick, T., Cohen, E.G.D., Dorfman, J. R., 1982, Fluctuations in a nonequilibrium staedy state: Basic equations, Phys. Rev. A, 26, 950-971.

61. Kirkpatrick, T., Cohen, E.G.D., Dorfman, 1982, J. R., Light scattering by a fluid in a nonequilibrium steady state. I. Small gradients, Phys. Rev. A, 26, 972-994.

62. Kirkpatrick, T., Cohen, E.G.D., Dorfman, J. R., 1982, Light scattering by a fluid in a nonequilibrium steady state. II. Large gradients, Phys. Rev. A, 26, 995-.1014.

63. Ronis, D., Procaccia, I., Oppenheim, I., 1979, Statistical mechanics of stationary states. III. Fluctuations in dense fluids with application of light scattering, Phys. Rev. A, 19, 1324-1339.

64. Ronis, D., Procaccia, I., 1982, Nonlinear resonant coupling between shear and heat fluctuations in fluids far from equilibrium, Phys. Rev. A, 26, 1812-1815

65. Beysens, D., Garrabos, Y., Zalczer, G., 1980, Experimental evidence for Brillouin asymmetry induced by a temperature gradient, Phy. Rev. Lett., 45, 403-406.

66. Law, B.M., Gammon, R.W., Sengers, J.V., 1988, Light-scattering observations of long-range correlations in a nonequilibrium fluid, Phys. Rev. Lett., 60, 1554-1557.

67. Segrè, P.N., Gammon, R.W., Sengers, J.V., Law, B.M., 1992, Rayleigh scattering in a liquid far from thermal equilibrium, Phys. Rev. A, 45, 714-724

68. Li, W.B., Segrè, P.N., Gammon, R.W., Sengers, J.V., 1994, Small-angle Rayleigh scattering from nonequilibrium fluctuations in liquids and liquid mixtures, Physica A, 204, 399-436. 
69. Rahman, A., Stillinger, F., 1974, Propagation of sound in water. A molecular-dynamics study, Phys. Rev. A, 10, 368-378.

70. Maxwell, J.C., 1867, On the dynamical theory of gases, Ph. Trans. Royal Soc. London, 157, 49-88

71. Peluso, F., 2003, JETC8 International conference on Thermodynamics Long-range collective dynamics and relaxation phenomena in a dual model of liquids, 113-122 (Barcelona, Spain, Sept. $2^{\text {nd }}-5^{\text {th }} 2003$ ).

72. Peluso, F., How does heat propagate in liquids? In preparation.

73. Mercier, J., 1956, De la pression de radiation dans le fluides, Jour. Phys. Rad., 17, 401-404.

74. Joyce, W.B., 1974, Radiation force and the classical mechanics of photons and phonons, Am. J. Phys., 43, 245255.

75. Brillouin, L., 1964, Tensors in Mechanics and Elasticity, pagg. 240-243 New York, Academic Press.

76. Smith, W.E., 1971, Generalization of the Boltzmann-Ehrenfest Adiabatic Theorem in Acoustics, J. Ac. Soc. Am., 50, 386-388

77. Gaeta, F.S., Ascolese, E., Tomicki, B., 1991, Radiation forces associated with heat propagation in nonisothermal systems, Phys. Rev. A, 44, 5003-5017

78. see for instance De Groot, S.R., Mazur, P., 1984, Non-equilibrum thermodynamics, New York, Dover pub. Inc..

79. Boltzmann, L., 1902, Leçons sur la théorie des gaz, Ch. 2-11, Paris, Gauthiers-Villars.

80. Cummins, H. Z., Gammon, R.W., 1966, Rayleigh and Brillouin scattering in liquids: the Landau-Placzek ratio, $J$. Chem. Phys., 44, 2785-2796.

81. Onsager, L., 1931, Reciprocal relations in irreversible processes I, Phys. Rev. 37, 405-426

82. Onsager, L., 1931, Reciprocal relations in irreversible processes II, Phys. Rev, 38, 2265-2279.

83. Peluso, F., Isochoric specific heat comparison in liquids between the Dual Model of Liquids and the Phonon Theory of Liquid Thermodynamics. In preparation.

84. Esposito, A., et al., 2019, Gravitational mass carried by sound waves, Phys. Rev. Lett., 122, 084501.

85. Albanese, C., Mantile, A., Peluso, F., 2002, A new thermoelastic model for thermal radiation pressure, Entropie, 239-240, 37-40

86. Herzfeld, K.F., Litovitz, T.A., 1959, Absorption and dispersion of ultrasonic waves, New York, Academic Press.

87. Nettleton, R.E., Compressional relaxation in liquids, J. Acoust. Soc. Am., 31, (1959) 557-567.

88. Nettleton, R.E, 1959, Thermodynamics of transport processes in liquids, Trans. Soc. Rheol., 3, 95-99.

89. Nettleton, R.E., 1960, Relaxation theory of thermal conduction in liquids, Phys. Fluids, 3, (1960), 216-225

90. A. Nicolis, R. Penco, 2018, Mutual interactions of phonons, rotons and gravity, Phys. Rev. B 97, 134516134553.

91. Dwayne Miller, R.J., 1991, Vibrational energy relaxation and structural dynamics of heme proteins, Ann. Rev. Phys. Chem., 42, 518.

92. Karplus, M. and McCammon, J.A., 1983, Dynamics of proteins: elements and function, Ann. Rev. Biochem., 53, 263.

93. McCammon, J.A., Gelin, B.R., Karplus, M., 1977 Dynamics of folded proteins, Nature, 267, 585. 


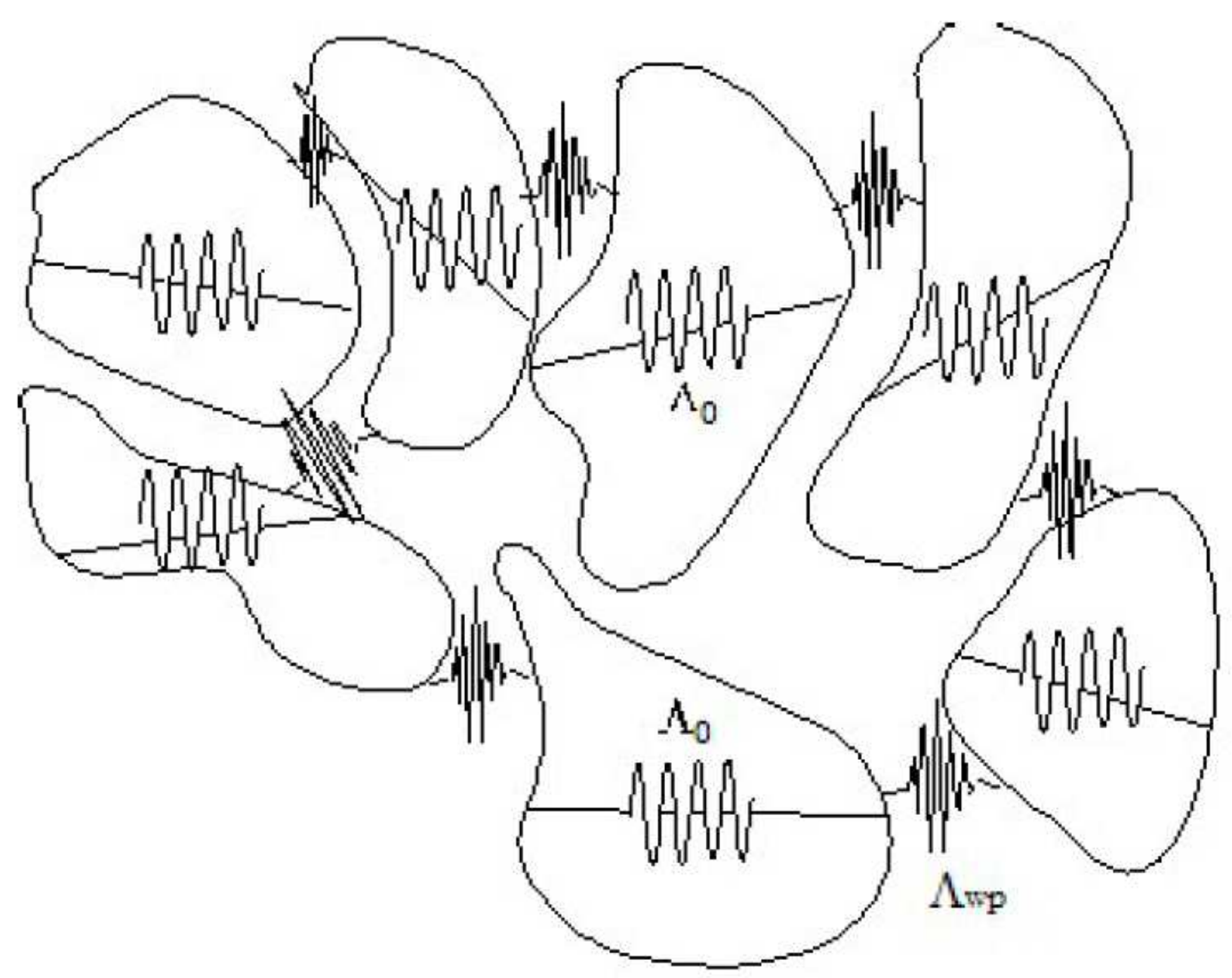

\section{Figure 1}

Dynamic solid-like structures fluctuating and interacting within the liquid global system at equilibrium. As far as thermal (elastic) perturbations propagate within such structures, they behave as in solids. Propagation velocity has then the values typical as those of the solid lattice, as found by Ruocco et al. [15] of about $3200 \mathrm{~m} / \mathrm{s}$ for the case of water. Average sizes of icebergs $\varangle \wedge 0 \bigotimes$ have been found of some nanometers. When perturbations cross the boundary between two solid-like aggregates, fth develops and energy and momentum are transmitted from one to the nearest-neighbour aggregate. This pictorial model of liquids at mesoscopic scale, on which the DML is based, reflects also what may be deduced from experiments performed with IXS techniques, able to observe liquids at such scale-lengths. In a solution solute particles may be considered as solid-like aggregates having elastic impedance different from that of the solvent. Energy and momentum exchanged between the two types of these dynamic icebergs produce a net effect resulting in the diffusion of the solute along the concentration gradient. If a 
temperature gradient is imposed externally, the net effect will depend on the prevailing flux of wave packets, which will give rise to thermal diffusion of one species with respect to the other.

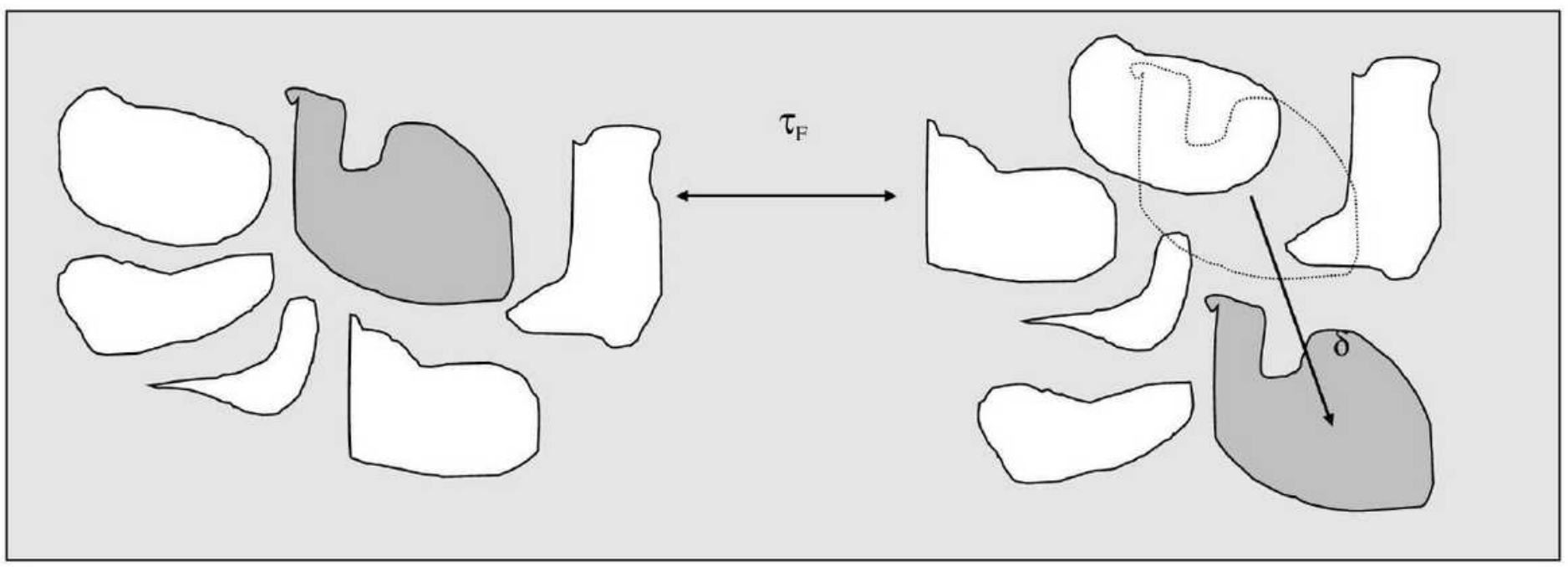

\section{Figure 2}

Illustration of a "wandering iceberg" jumping between two equilibrium positions in a liquid. The jump takes place after an average relaxation time lasting $\tau F$, during which the liquid particle moves by a distance $\delta$.

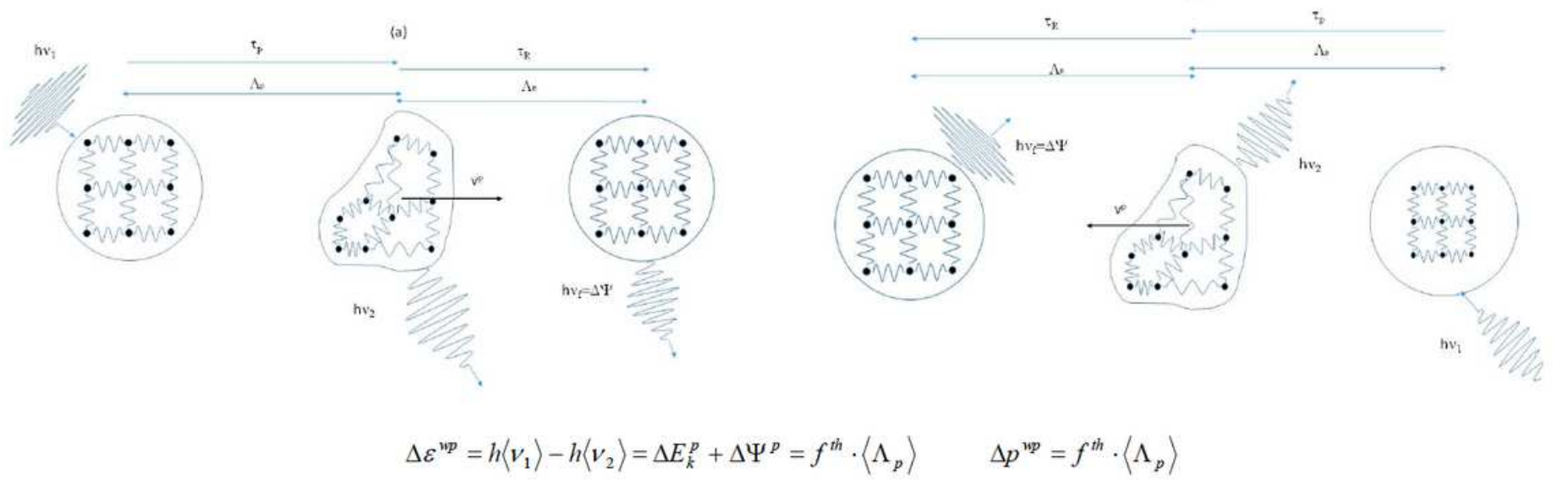

\section{Figure 3}

Schematic representation of wave-packets and liquid particles inelastic collisions. The event represented in (a), in which an energetic wave-packet transfers energy and momentum to a liquid particle, is commuted upon time reversal into the one represented in (b), where a liquid particle transfers energy and momentum to a wave-packet. The particle changes velocity and the frequency of wave-packet is shifted by the amount ( 1 1- $\mathrm{v} 2)$. The interaction lasts $\varangle \mathrm{tp} \rrbracket$ during which the particle moves by $₫ \wedge \mathrm{p} \rrbracket$; the relaxation lasts $₫ \tau R \rrbracket$ during which the particle moves by $\llbracket \wedge R \rrbracket$. Due to its time symmetry, this mechanism is assumed the equivalent of Onsager' reciprocity law at mesoscopic level. In a pure isothermal liquid energy and momentum exchanged among the icebergs are statistically equivalent, and no net effects are 
produced. Events of type a) will alternate with events of type b), to maintain the balance of the two energy pools unaltered. Besides, the macroscopic equilibrium will ensure also the mesoscopic equilibrium; events (a) and (b) will be equally probable along any direction, to have a zero average over time and space. On the contrary, if a symmetry breaking is introduced, as for instance a temperature or a concentration gradient, one type of event will prevail over the other along a preferential direction.

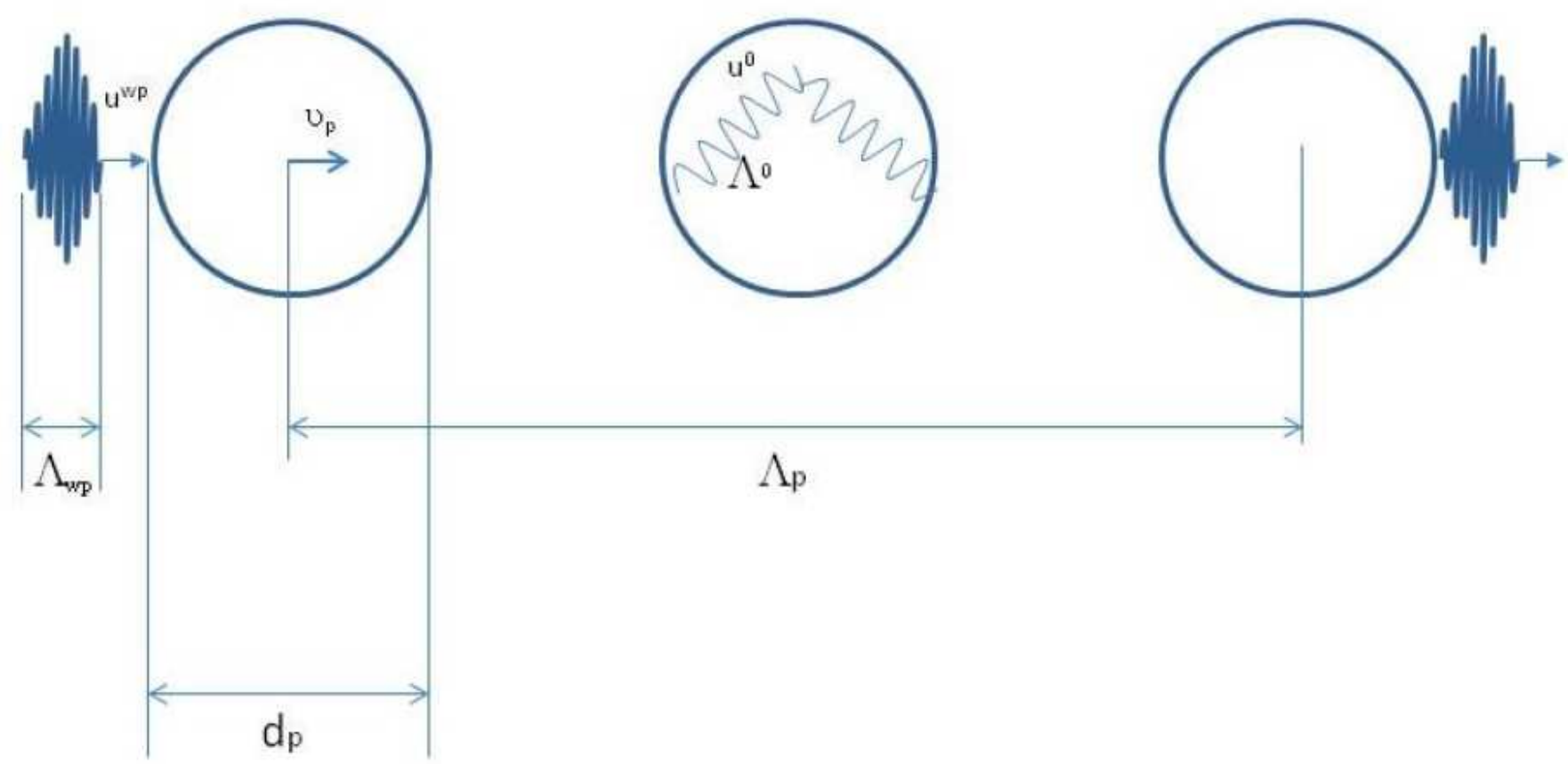

\section{Figure 4}

Close-up of the first part of the wave-packet - particle interaction shown in Figure 3a, during which the wave packet transfers energy and momentum to the liquid particle. $\Lambda w p$ is the extension of the wavepacket, and $d p$ that of the liquid particle. Once tp has elapsed and the liquid particle has travelled by $\Lambda p$, the particle relaxes the energy stored into internal DoF; then it travels by $\Lambda R$ during $\tau R$ (not shown in the figure above).

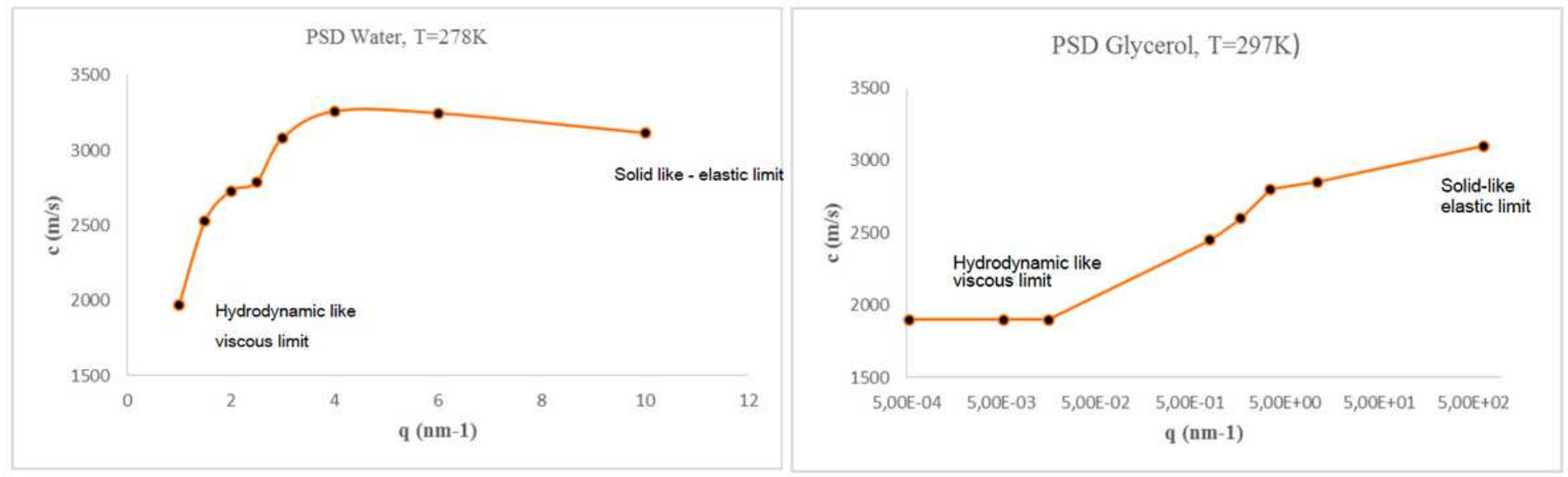

Figure 5 
Positive Sound Dispersion - PSD - for Water and Glycerol at the indicated temperature and momentum values. Data are from [23] for Water and from [10] for Glycerol. 